\title{
Decentralized Blocking Zeros and the Decentralized Strong Stabilization Problem
}

\author{
Konur A. Ünyelioğlu, A. Bülent Özgüler, and Ümit Özgüner
}

\begin{abstract}
This paper is concerned with a new system theoretic concept, decentralized blocking zeros, and its applications in the design of decentralized controllers for linear time-invariant finitedimensional systems. The concept of decentralized blocking zeros is a generalization of its centralized counterpart to multichannel systems under decentralized control. Decentralized blocking zeros are defined as the common blocking zeros of the main diagonal transfer matrices and various complementary transfer matrices of a given plant. As an application of this concept, we consider the decentralized strong stabilization problem (DSSP) where the objective is to stabilize a plant using a stable decentralized controller. It is shown that a parity interlacing property should be satisfied among the real unstable poles and real unstable decentralized blocking zeros of the plant for the DSSP to be solvable. That parity interlacing property is also sufficient for the solution of the DSSP for a large class of plants satisfying a certain connectivity condition. The DSSP is exploited in the solution of a special decentralized simultaneous stabilization problem, called the decentralized concurrent stabilization problem (DCSP). Various applications of the DCSP in the design of controllers for large-scale systems are also discussed.
\end{abstract}

\section{INTRODUCTION}

$\mathbf{I}_{\mathrm{d}}$ $\mathrm{N}$ this paper we introduce a new system theoretic concept, decentralized blocking zeros, and consider its applications in the design of decentralized controllers for linear timeinvariant finite-dimensional systems. Let $Z$ be an $N$-channel plant transfer matrix. By definition, a decentralized blocking zero $s_{0}$ of $Z$ is an element of the extended complex plane (complex plane appended by infinity) such that with some symmetric permutation of the block rows and columns $Z\left(s_{0}\right)$ becomes strictly upper block triangular.

Decentralized blocking zeros have significant roles in various decentralized control problems. The notion of decentralized blocking zeros determines the minimum unstable order of decentralized stabilizing controllers. Recall that in the centralized case, the minimum possible number of unstable poles of stabilizing controllers for a plant is determined by the odd distribution of real unstable poles between the real unstable blocking zeros [29, Theorem 5.3.1]. It is shown in this paper

Manuscript received November 6, 1992; revised April 29, 1994 and April 14, 1995. Recommended by Associate Editor, N. Gundes. This work was supported in part by National Science Foundation Grant INT-91011276, by Ford Motor Company under an unrestricted grant, and by Scientific and Technical Research Council of Turkey (TÜBİTAK) Grant TBAG-1016.

K. A. Ünyelioğlu and Ü. Özgüner are with the Department of Electrical Engineering, The Ohio State University, Columbus, OH 43210-1272 USA.

A. B. Özgüler is with the Department of Electrical and Electronics Engineering, Bilkent University, Bilkent, Ankara, 06533 Turkey.

IEEE Log Number 9414726. that, analogously, the minimum possible number of unstable poles of decentralized stabilizing controllers is determined by the odd distribution of real unstable plant poles between the real unstable decentralized blocking zeros. In particular, this result yields solvability conditions for the solution of the decentralized strong stabilization problem (DSSP) where the objective is to stabilize a plant using a stable decentralized controller [15]. Decentralized simultaneous stabilization problem is also related to the DSSP. We define a special decentralized simultaneous stabilization problem, called the decentralized concurrent stabilization problem (DCSP), which can be solved by transforming it to DSSP in a suitable auxiliary plant. A fundamental problem of decentralized control, namely the stabilization problem of a plant via stabilization of its subsystems, can be formulated as a DCSP. In this context, two general interconnection schemes are considered where the subsystems have transfer matrix or state-space representations.

Decentralized blocking zeros also have interpretations in terms of transmission zeros [18], pinned zeros [2], and a new concept, decentralized fixed zeros. The relations between these concepts are briefly discussed in the sequel. (See Remarks 2 and 3 and Corollary 2.)

The organization of the paper is as follows. The next section includes the notation and preliminaries. Section III considers the decentralized blocking zeros. In Section IV, we investigate the design of decentralized stabilizing controllers with minimum number of unstable poles. In particular, we examine the solution of the decentralized strong stabilization problem. Section V considers the solution of the decentralized concurrent stabilization problem. Section VI is devoted to some concluding remarks. Due to space limitations, the proofs of Lemmas 2-7 are omitted. They can be found in [27].

\section{Notation AND PRELIMinaries}

Let $\mathcal{C}$ and $\mathcal{R}$ denote the fields of complex and real numbers, respectively. By $\mathcal{C}_{e}$ and $\mathcal{R}_{e}$ we denote the extended complex and real numbers, i.e., the sets of complex and real numbers appended by $\infty$. We let $\operatorname{Re}(s)$ denote the real part of $s \in \mathcal{C}$ and define $\mathcal{C}_{+}=\{s \in \mathcal{C} \mid \operatorname{Re}(s) \geq 0\}, \mathcal{C}_{+e}=\mathcal{C}_{+} \cup\{\infty\}, \mathcal{R}_{+e}=$ $\mathcal{R}_{e} \cap \mathcal{C}_{+e}$. The set of proper real rational functions in the indeterminate $s$ is denoted by $\boldsymbol{P}$ and the set of stable proper real rational functions of $s$ is denoted by $\boldsymbol{S} . \boldsymbol{P}_{s}$ denotes the set of real rational functions whose denominator polynomials have no roots in $\mathcal{C}_{+}$. In other words, $\boldsymbol{P}_{s}$ is the set of stable (but not necessarily proper) rational functions. For a matrix 
$A$ over $S,\|A\|$ denotes the $H_{\infty}$ norm of $A$ defined in the usual way. By $I_{r}$, we denote the identity matrix of size $r$ and by $0_{r \times t}$, the zero matrix with $r$ rows and $t$ columns. The subscript is dropped if the size is clear from the context. The determinant of a square matrix $A$ is denoted by $\operatorname{det}(A)$ and the transpose of a matrix $B$ is denoted by $B^{\prime}$. For two real numbers $a, b, \min (a, b)$ is the minimum of these numbers. For all other undefined terminology and notation pertaining to the algebraic and topological structure of the ring $S$ and for matrices over $S$, we refer the reader to [29].

A complex number $s_{0}$ is called a blocking zero of $Z \in P^{p \times r}$ if $Z\left(s_{0}\right)=0$ [8], [9]. If $Z$ is stable, then the unstable blocking zeros are the unstable zeros of the smallest invariant factor (sif) of $Z$ over $S$. It is well known [29] that the blocking zeros of $Z$ are disjoint from the poles of $Z$. Let $\mathcal{S}_{1}$ and $\mathcal{S}_{2}$ be two finite collections of numbers in $\mathcal{R}_{+e}$, where in $\mathcal{S}_{1}$ some numbers may occur more than once. In case $\mathcal{S}_{1}$ and $\mathcal{S}_{2}$ are disjoint, we say that the ordered pair $\left(\mathcal{S}_{1}, \mathcal{S}_{2}\right)$ has parity interlacing property if there are an even number of elements from $\mathcal{S}_{1}$ between each pair of elements from $\mathcal{S}_{2}$. The terminology is borrowed from [32] in which $\mathcal{S}_{1}$ and $\mathcal{S}_{2}$ are, respectively, the poles (with multiplicity) and the blocking zeros of a transfer matrix. Note that, if $\mathcal{S}_{1}$ is the set of $\mathcal{R}_{+e}$ zeros with multiplicity of $a \in S$, then $a(s)$ takes the same sign at all elements $s \in \mathcal{S}_{2}$ if and only if $\left(\mathcal{S}_{1}, \mathcal{S}_{2}\right)$ has the parity interlacing property.

Let $A$ be a matrix over ring $\mathcal{C}$ or ring $\boldsymbol{P}$. Then, the notation $A=0$ is equivalent to saying that $A$ is identically zero, i.e., every entry of $A$ is the zero element of the associated ring. If $A$ is over $P$ then rank $A$ is the rank of $A$ over $P$ and rank $A(s)$ is the rank of $A(s)$ over $\mathcal{C}$ where $s \in \mathcal{C}_{+}$is such that it is not a pole of $A$.

Let $y=Z u$, and $y_{c}=Z_{c} u_{c}$ be the transfer matrix representations of a plant and a compensator, respectively, where $Z \in P^{p \times r}$ and $Z_{c} \in P^{r \times p}$. The plant and the compensator are interconnected according to the rules $u=v_{e}$ $-y_{c}, u_{c}=v_{c e}+y$, where $v_{e}$ and $v_{c e}$ denote some external inputs to the closed-loop system. The closed-loop system is well defined if $\left(I+Z Z_{c}\right)$ is nonsingular and $\left(I+Z Z_{c}\right)^{-1}$ is over $\boldsymbol{P}$, in which case the transfer matrix description for the closed-loop system is $\left[y^{\prime} y_{c}^{\prime}\right]^{\prime}=G\left[v_{e}^{\prime} v_{c e}^{\prime}\right]^{\prime}$, where

$$
G:=\left[\begin{array}{cc}
Z-Z Z_{c}\left(I+Z Z_{c}\right)^{-1} Z & -Z Z_{c}\left(I+Z Z_{c}\right)^{-1} \\
Z_{c}\left(I+Z Z_{c}\right)^{-1} Z & Z_{c}\left(I+Z Z_{c}\right)^{-1}
\end{array}\right]
$$

We say that $\left(Z, Z_{c}\right)$ is a stable pair if the closed-loop system is well defined and $G$ is a matrix over $S$ [29]. The following statements are equivalent by definition: $\left(Z, Z_{c}\right)$ is a stable pair; $Z_{c}$ stabilizes $Z ; Z_{c}$ is a stabilizing controller for $Z$; the closed-loop system associated with the pair $\left(Z, Z_{c}\right)$ is stable.

Let a bicoprime fractional representation of $Z$ over $S$ be given by

$$
Z=P Q^{-1} R \text {. }
$$

Also let $Z_{c}=P_{c} Q_{c}^{-1}$ be a right coprime fractional representation of $Z_{c}$ over $S$. Then, $\left(Z, Z_{c}\right)$ is a stable pair if and only if the matrix

$$
\left[\begin{array}{cc}
Q & R P_{c} \\
-P & Q_{c}
\end{array}\right]
$$

is unimodular over $\boldsymbol{S}$ [7] or, equivalently, invertible over $\boldsymbol{S}$. In particular, if $Z_{c}$ is a matrix over $S$, then the matrix (2) is unimodular if and only if so is the matrix $Q+R Z_{c} P$.

We now state a main result on the determination of a stabilizing controller with minimum number of unstable poles for $Z$ where we assume that $Z$ is not identically zero.

Let $\sigma_{1}, \sigma_{2}, \cdots, \sigma_{t}$ denote the $\mathcal{R}_{+e^{-b l o c k i n g ~ z e r o s ~ o f ~} Z}$ arranged in ascending order. Let $\eta_{i}$ denote the number of $\mathcal{R}_{+}$poles of $Z$ counted with multiplicities in the interval $\left(\sigma_{i}, \sigma_{i+1}\right), i \in\{1,2, \cdots, t-1\}$. Also let $\eta$ be the number of odd integers in the set $\left\{\eta_{1}, \cdots, \eta_{t-1}\right\}$. The following result can be proven using Theorem 5.3.1 of [29].

Theorem 1:

i) Every stabilizing controller $Z_{c}$ for $Z$ has at least $\eta$ poles in $\mathcal{C}_{+}$with multiplicities.

ii) a) Given any integer $n \geq \eta$ where $n-\eta$ is an even number, there exists a stabilizing controller $Z_{c}$ for $Z$ which has exactly $n$ poles in $\mathcal{C}_{+}$with multiplicities.

b) Given any integer $n \geq \eta$ where $n-\eta$ is an odd number, there exists a stabilizing controller $Z_{c}$ for $Z$ which has exactly $n$ poles in $\mathcal{C}_{+}$with multiplicities if and only if $\sigma_{1} \neq 0$ or $\sigma_{t} \neq \infty$.

The strong stabilization problem is defined as determining a stable controller $Z_{c}$, i.e., a controller having all entries over $S$, such that $\left(Z, Z_{c}\right)$ is stable [32], [29]. From Theorem 1 we conclude that the strong stabilization problem is solvable if and only if there are an even number of poles of $Z$ between each pair of its blocking zeros; equivalently, the set of unstable real poles with multiplicity of $Z$ and the set of unstable real blocking zeros of $Z$ have the parity interlacing property.

Let $Z=\left[Z_{i j}\right], Z_{i j} \in \boldsymbol{P}^{p_{i} \times r_{j}}, i, j \in \boldsymbol{N}$, be an $N$ channel plant. We denote by $\boldsymbol{N}$ the ordered set of integers $\{1$, $2, \cdots, N\}$. The decentralized stabilization problem (DSP) is defined as determining a controller $Z_{c}=\operatorname{diag}\left\{Z_{c 1}, \cdots, Z_{c N}\right\}$ where $Z_{c i} \in \boldsymbol{P}^{\boldsymbol{r}_{i} \times p_{i}}, i \in \boldsymbol{N}$, such that $\left(Z, Z_{c}\right)$ is stable. If there exists such a $Z_{c}$ we say that $Z_{c}$ solves DSP for $Z$. By definition, this is equivalent to saying that $Z_{c}$ is a decentralized stabilizing controller for $Z$. Let the matrices $P$ and $R$ in (1) be partitioned as $P=\left[P_{1}^{\prime} \cdots P_{N}^{\prime}\right]^{\prime}$ and $R=\left[R_{1} \cdots R_{N}\right]$, where $P_{i} Q^{-1} R_{j}=Z_{i j}$. DSP for $Z$ is solvable if and only if $Z$ has no unstable decentralized fixed modes [31]. An equivalent solvability condition can be given in terms of the fractional representation above as follows. For a proper subset $\mathcal{L}$ of $\boldsymbol{N}$ define $\boldsymbol{N}-\mathcal{L}$ to be the complement of $\mathcal{L}$ in $\boldsymbol{N}$. For a set $\mathcal{K}$ of positive indices $R_{\mathcal{K}}$ denotes the submatrix of $R$ consisting of $R_{i}$ 's with indices in $\mathcal{K} . P_{\mathcal{K}}$ is defined similarly.

Lemma 1: DSP is solvable if and only if for every proper subset $\mathcal{L}$ of $\boldsymbol{N}$ it holds that

$$
\operatorname{rank}\left[\begin{array}{cc}
Q & R_{\mathcal{L}} \\
-P_{\boldsymbol{N}-\mathcal{L}} & 0
\end{array}\right](s) \geq \operatorname{size}(Q), \forall s \in \mathcal{C}_{+} .
$$


A proof of Lemma 1 can be found in [10, Chap. 4] which is based on an earlier result developed for two-channel systems in [6]. Various algebraic characterizations of decentralized stabilizing controllers are available in the literature where alternative construction techniques for the solution of DSP are presented. (See, e.g., [10], [11], [4], [13], [23].)

The system $\left(P_{N_{-\mathcal{L}}}, Q, R_{\mathcal{L}}\right)$ is called a complementary subsystem of $(P, Q, R)$ [3]. The subsystem transfer matrix $Z_{N_{-\mathcal{L}, \mathcal{L}}}:=P_{N_{-\mathcal{L}}} Q^{-1} R_{\mathcal{L}}$ is called a complementary transfer matrix of $Z$. The plant $Z$ is called strongly connected if all its complementary transfer matrices are nonzero, i.e., $Z_{\boldsymbol{N}_{-\mathcal{L}, \mathcal{L}}} \neq$ 0 , for all proper subsets $\mathcal{L}$ of $\boldsymbol{N}$ [3].

In the sequel, bicoprime fractional representations of the plant transfer matrices are extensively used. There are several motivations for dealing with bicoprime rather than left or right coprime (doubly coprime) fractional representations [7]. On the contrary to doubly coprime representations, a bicoprime representation of a plant transfer matrix is readily available from its state-space realization. For example, if $(C, A, B)$ is a stabilizable and detectable realization of a transfer matrix $Z$ such that $Z=C(s I-A)^{-1} B$ then $Z=P Q^{-1} R$ is a bicoprime fraction of $Z$ over $S$ where $P:=C(1 / s+1), Q:=$ $(s I-A)(1 / s+1)$, and $R:=B$. Using such natural bicoprime representations enables us to give some of the analysis results in the sequel in terms of the original system matrix structure of the plant. Doubly coprime representations, on the other hand, are easier to manipulate in many instances. Therefore, in the proofs of various technical lemmas we sometimes utilize the doubly coprime representations for convenience.

\section{DECENTRALIZED BlockING ZEROS}

Let $Z$ be the transfer matrix of an $N$-channel system $(N>1)$ so that it is in the partitioned form $Z=\left[Z_{i j}\right]$, where $Z_{i j} \in \boldsymbol{P}^{p_{i} \times r_{j}}, i, j \in \boldsymbol{N}$ such that $\sum_{i=1}^{N} p_{i}=p$ and $\Sigma_{i=1}^{N} r_{i}=r$. Let a bicoprime fractional representation of $Z$ be given by

$$
Z=\left[P_{1}^{\prime} \cdots P_{N}^{\prime}\right]^{\prime} Q^{-1}\left[R_{1} \cdots R_{N}\right]
$$

for some $P_{i} \in S^{p_{i} \times q}, R_{i} \in S^{q \times r_{i}}, i=1, \cdots, N$, and $Q \in S^{q \times q}$ so that $Z_{i j}=P_{i} Q^{-1} R_{j}, i, j=1, \cdots, N$. An element $s$ of $\mathcal{C}_{e}$ is called a decentralized blocking zero of $Z$ if, when evaluated at $s$, all the block entries of plant transfer matrix below the main diagonal band and those in the main diagonal band become zero after a suitable symmetric permutation of the block rows and columns. More precisely, $s$ is a decentralized blocking zero of $Z$ if and only if for some permutation $\left\{i_{1}, \cdots, i_{N}\right\}$ of $N$ the following holds: $Z_{i_{k} i_{l}}(s)=$ $0, k=1, \cdots, N, l=1, \cdots, k$. The set of decentralized blocking zeros of $Z$ is denoted by $\mathcal{S}_{Z}$. It follows that

$$
\mathcal{S}_{Z}=\left\{s \in \mathcal{C}_{e} \mid \text { There exists a permutation }\left\{i_{1}, i_{2}, \cdots, i_{N}\right\}\right.
$$

of $\boldsymbol{N}$ such that

$$
\left.\left[\begin{array}{ccccc}
Z_{i_{1} i_{1}} & 0 & 0 & \cdots & 0 \\
Z_{i_{2} i_{1}} & Z_{i_{2} i_{2}} & 0 & \cdots & 0 \\
Z_{i_{3} i_{1}} & Z_{i_{3} i_{2}} & Z_{i_{3} i_{3}} & \cdots & 0 \\
\vdots & \vdots & \vdots & \ddots & 0 \\
Z_{i_{N} i_{1}} & Z_{i_{N} i_{2}} & Z_{i_{N} i_{3}} & \cdots & Z_{i_{N} i_{N}}
\end{array}\right](s)=0\right\}
$$

For convenience, in the case $N=1$ (the centralized case), we define the decentralized blocking zeros as the centralized blocking zeros.

An equivalent description for the set $\mathcal{S}_{Z}$ can be given as follows. Define $\mathcal{S}_{Z}^{\text {diag }}=\left\{s \in \mathcal{C}_{e} \mid Z_{i i}(s)=0, i \in N\right\}$

$$
\begin{aligned}
\mathcal{S}_{Z}^{\text {comp }}= & \left\{s \in \mathcal{C}_{e} \mid\right. \text { There exists a permutation } \\
& \left\{i_{1}, \cdots, i_{N}\right\} \text { of } N \text { such that } \\
& s \text { is a blocking zero of all the } \\
& \text { complementary transfer matrices below: } \\
& {\left[Z_{i_{2} i_{1}}^{\prime} Z_{i_{3} i_{1}}^{\prime} \cdots Z_{i_{N} i_{1}}^{\prime}\right]^{\prime},\left[\begin{array}{cc}
Z_{i_{3} i_{1}} & Z_{i_{3} i_{2}} \\
\vdots & \vdots \\
Z_{i_{N} i_{1}} & Z_{i_{N} i_{2}}
\end{array}\right], \cdots } \\
& {\left.\left[Z_{i_{N} i_{1}} Z_{i_{N} i_{2}} \cdots Z_{i_{N} i_{N-1}}\right]\right\} }
\end{aligned}
$$

It easily follows that

$$
\mathcal{S}_{Z}=\mathcal{S}_{Z}^{\text {diag }} \cap \mathcal{S}_{Z}^{\mathrm{comp}}
$$

That is, every decentralized blocking zero is a common blocking zero of all the main diagonal transfer matrices and various complementary transfer matrices. In the simplest case of two channels, these alternative descriptions yield the following expressions for $\mathcal{S}_{Z}$

$$
\begin{aligned}
\mathcal{S}_{Z}= & \left\{s \in \mathcal{C}_{e} \mid Z_{11}(s)=0, Z_{21}(s)=0, \text { and } Z_{22}(s)=0\right\} \\
& \cdot \cup\left\{s \in \mathcal{C}_{e} \mid Z_{22}(s)=0, Z_{12}(s)=0,\right. \text { and } \\
& \left.\cdot Z_{11}(s)=0\right\} \\
= & \left\{s \in \mathcal{C}_{e} \mid Z_{11}(s)=0 \text { and } Z_{22}(s)=0\right\} \\
& \cdot \cap\left\{s \in \mathcal{C}_{e} \mid Z_{21}(s)=0 \text { or } Z_{12}(s)=0\right\}
\end{aligned}
$$

Note that, any (centralized) blocking zero is clearly a decentralized blocking zero and also $\mathcal{S}_{Z}$ can be a much larger set than the set $\left\{s \in \mathcal{C}_{e} \mid Z(s)=0\right\}$ of blocking zeros.

The fact that the $\mathcal{C}_{+e}$ centralized blocking zeros are disjoint with the poles of $Z$ does not directly extend to the decentralized blocking zeros. For example, consider the $2 \times 2$ (two scalar input-two scalar output) transfer matrix

$$
Z=\left[\begin{array}{cc}
\frac{s}{s-1} & \frac{1}{s} \\
\frac{s}{s-1} & \frac{s}{s-1}
\end{array}\right] .
$$

The poles are $\{0,1,1\}$ and the only decentralized blocking zero is $\{0\}$. The common element 0 is easily seen to be a decentralized fixed mode of $Z$ [16]. 
Lemma 2: Consider the $N$-channel plant transfer matrix $Z=\left[Z_{i j}\right]$. If $s_{0} \in \mathcal{S}_{Z} \cap \mathcal{C}_{+e}$ is a pole of $Z$ then it is a decentralized fixed mode.

Remark 1: Consider a permutation $P=\left\{i_{1}, \cdots, i_{N}\right\}$ of $\boldsymbol{N}$ and $j \in \boldsymbol{N}$. Then, $s_{0} \in \mathcal{C}_{+e}$ is called an unstable invariant zero associated with the $l$ th invariant factor of system $\left(\left[P_{i_{j}}^{\prime} \cdots P_{i_{N}}^{\prime}\right]^{\prime}, Q,\left[R_{i_{1}} \cdots R_{i_{j}}\right]\right)$ where $1 \leq l \leq \operatorname{rank}\left[P_{i_{j}}^{\prime} \cdots\right.$ $\left.P_{i_{N}}^{\prime}\right]^{\prime} Q^{-1}\left[R_{i_{1}} \cdots R_{i_{j}}\right]+q$, if

$$
\operatorname{rank}\left[\begin{array}{cccc}
Q & R_{i_{1}} & \cdots & R_{i_{j}} \\
-P_{i_{j}} & 0 & \cdots & 0 \\
\vdots & \vdots & & \vdots \\
-P_{i_{N}} & 0 & \cdots & 0
\end{array}\right]\left(s_{0}\right)<l .
$$

Let $\boldsymbol{N}_{P}$ be a subset of $\boldsymbol{N}$ such that $j \in \boldsymbol{N}_{P}$ if and only if $\left[P_{i_{j}}^{\prime} \cdots P_{i_{N}}^{\prime}\right]^{\prime} Q^{-1}\left[R_{i_{1}} \cdots R_{i_{j}}\right] \neq 0$. The following result can be proven using various lemmas in [27]: $s \in \mathcal{C}_{+e}$ is a decentralized blocking zero of a plant $Z$ which has no $\mathcal{C}_{+}$decentralized fixed modes if and only if there exists a permutation $P=\left\{i_{1}, \cdots, i_{N}\right\}$ of $\boldsymbol{N}$ such that $s$ is a common invariant zero associated with the $(q+1)$-st invariant factor of systems $\left(\left[P_{i_{j}}^{\prime} \cdots P_{i_{N}}^{\prime}\right]^{\prime}, Q,\left[R_{i_{1}} \cdots R_{i_{j}}\right]\right), j \in \boldsymbol{N}_{P}$. (If $\boldsymbol{N}_{P}$ is empty set, observe that $Z$ can be transformed to a strictly upper triangular structure via symmetric column and row permutations. Consequently, every $s \in \mathcal{C}_{e}$ is a decentralized blocking zero of $Z$.)

Remark 2: We will now define decentralized fixed zeros and consider their relation with the decentralized blocking zeros.

Consider the $N$-channel plant $Z=\left[Z_{i j}\right]$ and its bicoprime fractional representation (4) over $S$. For any $i \in N$ let a collection of $N-1$ controllers be given

$$
\begin{aligned}
\left(Z_{c 1}, \cdots, Z_{c(i-1)}, Z_{c(i+1)}, \cdots, Z_{c N}\right) & \\
\in & P^{r_{1} \times p_{1}} \times \cdots \times \boldsymbol{P}^{r_{i-1} \times p_{i-1}} \times \boldsymbol{P}^{r_{i+1} \times p_{i+1}} \\
& \times \cdots \boldsymbol{P}^{r_{N} \times p_{N}} .
\end{aligned}
$$

Define

$$
\begin{aligned}
& \Phi_{i}\left(Z_{c 1}, \cdots, Z_{c(i-1)}, Z_{c(i+1)}, \cdots, Z_{c N}\right) \\
& \left.=P_{i} 0, \cdots 00, \cdots 0\right] \mathcal{X}^{-1}\left[R_{i}^{\prime} 0 \cdots 00 \cdots 0\right]^{\prime}
\end{aligned}
$$

where (see the equation at the bottom of the page) and it is assumed that (8) is well defined and $P_{c j} Q_{c j}^{-1}=Z_{c j}, j=$
$1, \cdots, N, j \neq i$ are coprime representations. Now, define

$$
\begin{aligned}
\mathcal{Z}_{c i}= & \left(Z_{c 1}, \cdots, Z_{c(i-1)}, Z_{c(i+1)}, \cdots, Z_{c N}\right) \\
& \in \boldsymbol{P}^{r_{1} \times p_{1}} \times \cdots \times \boldsymbol{P}^{r_{i-1} \times p_{i-1}} \times \boldsymbol{P}^{r_{i+1} \times p_{i+1}} \\
& \times \cdots \boldsymbol{P}^{r_{N} \times p_{N}} \mid \\
& \Phi_{i}\left(Z_{c 1}, \cdots, Z_{c(i-1)}, Z_{c(i+1)}, \cdots, Z_{c N}\right)
\end{aligned}
$$

is a bicoprime fractional representation .

In other words, $\mathcal{Z}_{c i}$ is the set of all controllers which, when applied around the channels $1, \cdots, i-1, i+1, \cdots, N$, make the resulting single-channel system around channel $i$ stabilizable and detectable. From [23, Remark and Theorem 3.2] we have the following result: For any $\operatorname{diag}\left\{Z_{c 1}, \cdots, Z_{c N}\right\}$ solving DSP for $Z,\left(Z_{c 1}, \cdots, Z_{c(i-1)}, Z_{c(i+1)}, \cdots, Z_{c N}\right) \in \mathcal{Z}_{c i}$, for each $i \in \boldsymbol{N}$. Conversely, for a fixed $i \in \boldsymbol{N}$, consider any $\left(Z_{c 1}, \cdots, Z_{c(i-1)}, Z_{c(i+1)}, \cdots, Z_{c N}\right) \in \mathcal{Z}_{c i}$. Then, there exists $Z_{c i}$ such that $\operatorname{diag}\left\{Z_{c 1}, \cdots, Z_{c(i-1)}, Z_{c i}, Z_{c(i+1)}, \cdots, Z_{c N}\right\}$ solves DSP for $Z$.

Let $i \in N$ be fixed. A number $s \in \mathcal{C}_{+e}$ is called a decentralized fixed zero of channel i of the $N$-channel system $Z$ if $s$ is a blocking zero of $\Phi_{i}\left(Z_{c 1}, \cdots, Z_{c(i-1)}, Z_{c(i+1)}, \cdots, Z_{c N}\right)$ for every element $\left(Z_{c 1}, \cdots, Z_{c(i-1)}, Z_{c(i+1)}, \cdots, Z_{c N}\right)$ of $\mathcal{Z}_{c i}$. That is, $s$ is called a decentralized fixed zero of channel $i$ of $Z$, if $s$ appears as a blocking zero of channel $i$ in the partially closed-loop system resulting from the application of every $N-1$ local controllers around the other channels which yield that the single channel system around channel $i$ is stabilizable and detectable. Note that for some local controllers in $\mathcal{Z}_{c i}$, an element $s$ of $\mathcal{C}_{+e}$ can appear as a blocking zero at channel $i$ in the partially closed-loop system regardless of $s$ is a decentralized fixed zero or not. If $s$ is not a decentralized fixed zero, however, it can always be removed by the application of some other local controllers in $\mathcal{Z}_{c i}$ [27] (see also [26]).

The following statement establishes a relation between the decentralized blocking zeros and decentralized fixed zeros. It states that $s \in \mathcal{C}_{+e}$ is an unstable decentralized blocking zero of a plant $Z$ which has no unstable decentralized fixed modes if and only if $s$ is not a pole of $Z$ and is a common decentralized fixed zero of all channels. A proof of the statement can be found in [27].

Define, for $i \in N$

$$
\begin{aligned}
\mathcal{F}_{i}= & \left\{s \in \mathcal{C}_{+e} \mid s\right. \text { is a decentralized fixed zero } \\
& \text { of channel } i\} .
\end{aligned}
$$

$$
\mathcal{X}:=\left[\begin{array}{ccccccc}
Q & R_{1} P_{c 1} & \cdots & R_{i-1} P_{c(i-1)} & R_{i+1} P_{c(i+1)} & \cdots & R_{N} P_{c N} \\
-P_{1} & Q_{c 1} & \cdots & 0 & 0 & \cdots & 0 \\
\vdots & \vdots & \ddots & \vdots & \vdots & & \vdots \\
-P_{i-1} & 0 & \cdots & Q_{c(i-1)} & 0 & \cdots & 0 \\
-P_{i+1} & 0 & \cdots & 0 & Q_{c(i+1)} & \cdots & 0 \\
\vdots & \vdots & \vdots & \vdots & \vdots & \ddots & \vdots \\
-P_{N} & 0 & \cdots & 0 & 0 & \cdots & Q_{c N}
\end{array}\right]
$$


Let the $N$-channel system $Z$ have no $\mathcal{C}_{+}$decentralized fixed modes. Then, $\mathcal{S}_{Z} \cap \mathcal{C}_{+e}=\mathcal{F}$ where $\mathcal{F}:=\left\{s \in \mathcal{C}_{+e} \mid s\right.$ is not a pole of $Z$ and $s \in \cap_{i \in N} \mathcal{F}_{i}$.

Remark 3: An interpretation of decentralized blocking zeros can also be given in terms of the pinned zero concept introduced by Bristol [2]. An element $s_{0} \in \mathcal{C}$ is called a $k$-pinned zero of $Z \in P^{N \times N}$ if there exists a subset $J:=\left\{j_{1}, \cdots, j_{k}\right\}$ of distinct elements of $\boldsymbol{N}$ such that $\left(s-s_{0}\right)$ divides every $k \times k$ minor of the submatrix of $Z(s)$ consisting of its rows with indices in $J$. Assume that $Z$ is partitioned such that $Z_{i j}, i, j \in \boldsymbol{N}$ are all scalars. Let $s_{0}$ be a decentralized blocking zero of $Z$ where for some permutation $\left\{i_{1}, \cdots, i_{N}\right\}$ of $\boldsymbol{N}$ the following holds: $Z_{i_{k} i_{l}}\left(s_{0}\right)=0, k=1, \cdots, N, l=$ $1, \cdots, k$. It is easy to see that $s_{0}$ is a one-pinned zero of $Z$ associated with row $i_{N}$, a two-pinned zero associated with rows $i_{N}$ and $i_{N-1}, \cdots$ and a $N$-pinned zero associated with rows $i_{N}, i_{N-1}, \cdots, i_{1}$. The converse, however, is not true in general: an arbitrary pinned zero of a transfer matrix is not necessarily a decentralized blocking zero.

\section{Least Number of Unstable Controller Poles}

In this section we consider the construction of decentralized stabilizing controllers with minimum number of unstable poles. As a particular case, we examine the solution of decentralized strong stabilization problem. (See [28] for an alternative approach to the solution of this problem.) In terms of the notation of Section II, a more precise definition of decentralized strong stabilization problem can be given as follows.

Decentralized Strong Stabilization Problem (DSSP): Let $Z=\left[Z_{i j}\right], Z_{i j} \in \boldsymbol{P}^{p_{i} \times r_{j}}, i, j \in \boldsymbol{N}$ be the transfer matrix of a given plant. Determine (stable) local controllers $Z_{c i} \in$ $S^{r_{i} \times p_{i}}, i \in \boldsymbol{N}$ such that the pair $\left(Z, \operatorname{diag}\left\{Z_{c 1}, \cdots, Z_{c N}\right\}\right)$ is stable.

We first investigate how dynamic feedback at one channel affects the unstable decentralized blocking zeros. This is done for feedbacks which do not introduce any unstable decentralized fixed mode to the resulting $(N-1)$-channel system. For any such feedback, Lemma 3 below states that the set of unstable decentralized blocking zeros of the resulting $(N-1)$-channel system essentially includes the set of unstable decentralized blocking zeros of the original $N$-channel system. Lemma 3 will be employed in the proof of Theorem 2-i) below.

Lemma 3: Let an $N$-channel transfer matrix $Z=\left[Z_{i j}\right]$ have no $\mathcal{C}_{+}$decentralized fixed modes and have the bicoprime fractional representation (4). Define $L=N-1$. Let $Z_{c N}=$ $P_{c N} Q_{c N}^{-1} \in P^{r_{N} \times p_{N}}$ be a compensator at the $N$ th channel of (4), for a right coprime pair of matrices $\left(Q_{\mathrm{c} N}, P_{\mathrm{c} N}\right)$ over $S$, such that the resulting fraction

$$
\hat{Z}\left(Z_{c N}\right):=\left[\begin{array}{cc}
P_{1} & 0 \\
\vdots & \vdots \\
P_{L} & 0
\end{array}\right]\left[\begin{array}{cc}
Q & R_{N} P_{c N} \\
-P_{N} & Q_{c N}
\end{array}\right]^{-1}\left[\begin{array}{ccc}
R_{1} & \cdots & R_{L} \\
0 & \cdots & 0
\end{array}\right]
$$

of the $L$-channel system is a bicoprime fraction and (if $L>1) \hat{Z}\left(Z_{c N}\right)$ has no $\mathcal{C}_{+}$decentralized fixed modes. Then, $\mathcal{S}_{Z} \cap \mathcal{C}_{+e} \subset \mathcal{S}_{\hat{Z}\left(Z_{c N}\right)} \cap \mathcal{C}_{+e}$ where $\mathcal{S}_{\hat{Z}\left(Z_{c N}\right)}$ is the set of decentralized blocking zeros of $\hat{Z}\left(Z_{c N}\right)$.
Consider the following assumptions:

A1) $Z$ is strongly connected.

A2) $\operatorname{rank} Z_{i j} \geq 2$ or $\operatorname{rank} Z_{j i} \geq 2, \forall i, j \in \boldsymbol{N}, i \neq j$.

Assertion (10) will be utilized in the design part of Theorem 2 below. Assumption A1) can be assumed to hold with no loss of generality; if it fails then $Z$ can be decomposed into its strongly connected components and the problem of constructing a least unstable decentralized stabilizing controller and DSSP can be considered for each strongly connected subsystem independently. Assumption A2) is made because of technical reasons. See Section IV-B for a detailed discussion on this assumption.

Note that $\mathcal{S}_{Z}$ is a finite set if and only if there does not exist a permutation $\left\{i_{1}, \cdots, i_{N}\right\}$ of $N$ where

$$
\left[\begin{array}{ccccc}
Z_{i_{1} i_{1}} & 0 & 0 & \cdots & 0 \\
Z_{i_{2} i_{1}} & Z_{i_{2} i_{2}} & 0 & \cdots & 0 \\
Z_{i_{3} i_{1}} & Z_{i_{3} i_{2}} & Z_{i_{3} i_{3}} & \cdots & 0 \\
\vdots & \vdots & \vdots & \ddots & 0 \\
Z_{i_{N} i_{1}} & Z_{i_{N} i_{2}} & Z_{i_{N} i_{3}} & \cdots & Z_{i_{N} i_{N}}
\end{array}\right]
$$

is identically zero. It is easy to see that if $Z$ is strongly connected then $\mathcal{S}_{Z}$ is a finite set. Define $\Psi=\mathcal{S}_{Z} \cap \mathcal{R}_{+e}$ which is the set of decentralized blocking zeros of $Z$ lying in the extended right-half real line. Let $\sigma_{1}, \sigma_{2}, \cdots, \sigma_{t}$ denote the elements of $\Psi$ arranged in the ascending order. Also let $\eta_{i}$ denote the number of $\mathcal{R}_{+}$poles of $Z$ counted with multiplicities in the interval $\left(\sigma_{i}, \sigma_{i+1}\right), i \in\{1,2, \cdots, t-$ 1 \}. Define $\eta$ to be the number of odd integers in the set $\left\{\eta_{1}, \cdots, \eta_{t-1}\right\}$.

The following lemma is a key result which is used in the proof of Theorem 2-ii) below. Briefly, it says for those plants satisfying (10) that given any nonnegative integer $n_{N} \leq \eta$ one can construct a local controller around any fixed but otherwise arbitrary channel (the $N$ th channel below without loss of generality) which has $n_{N}$ poles in $\mathcal{C}_{+}$with multiplicities and ensures that DSP for the resulting $L:=N-1$ channel plant $\hat{Z}\left(Z_{c N}\right)$ is solvable and satisfies an appropriate interlacing property between the set of real unstable poles and the set of real unstable decentralized blocking zeros.

Lemma 4: Let an $N$-channel transfer matrix $Z=\left[Z_{i j}\right]$ have no $\mathcal{C}_{+}$decentralized fixed modes and have the bicoprime fractional representation (4). Assume that $Z$ satisfies (10). Let a nonnegative integer $n_{N} \leq \eta$ be given. There exists $Z_{c N}=P_{c N} Q_{c N}^{-1} \in P^{r_{N} \times p_{N}}$ for a right coprime pair of matrices $\left(Q_{c N}, P_{c N}\right)$ over $\boldsymbol{S}$ such that:

a) $Z_{c N}$ has $n_{N} \mathcal{C}_{+}$poles counted with multiplicities.

b) The fraction (9) of $\hat{Z}\left(Z_{c N}\right)$ is bicoprime.

c) Denoting by $\mathcal{S}_{\hat{Z}\left(Z_{c N}\right)}$ the set of decentralized blocking zeros of $\hat{Z}\left(Z_{c N}\right)$ and letting $\bar{\sigma}_{1}, \bar{\sigma}_{2}, \cdots, \bar{\sigma}_{\bar{t}}$ denote the elements of

$$
\Psi_{L}(\hat{Z}):=\mathcal{S}_{\hat{Z}\left(Z_{c N}\right)} \cap \mathcal{R}_{+e}
$$

arranged in the ascending order and denoting by $\bar{\eta}_{i}$ the number of $\mathcal{R}_{+}$poles of $\hat{Z}\left(Z_{c N}\right)$ counted with multiplicities in the interval $\left(\bar{\sigma}_{i}, \bar{\sigma}_{i+1}\right), i \in\{1,2, \cdots, \bar{t}-$ $1\}$, it holds that $\bar{\eta}=\eta-n_{N}$ where $\bar{\eta}$ is the number of odd integers in the sequence $\bar{\eta}_{1}, \cdots, \bar{\eta}_{\bar{t}-1}$. 
d) (If $L>1$ ) DSP for $\hat{Z}\left(Z_{c N}\right)$ is solvable, $\hat{Z}\left(Z_{c N}\right)$ is strongly connected and satisfies rank $\hat{Z}_{i j} \geq 2$ or rank $\hat{Z}_{j i} \geq 2 \forall i, j \in \boldsymbol{L}, i \neq j$ where $\hat{Z}_{i j} \in \boldsymbol{P}^{p_{i} \times r_{j}}$ denotes the $(i, j)$ th submatrix of $\hat{Z}\left(Z_{c N}\right)$.

We can now state the main result of this paper.

Theorem 2: Let an $N$-channel transfer matrix $Z=\left[Z_{i j}\right]$ have no $\mathcal{C}_{+}$decentralized fixed modes and have the bicoprime fractional representation (4).

i) Every decentralized stabilizing controller $Z_{c}=\operatorname{diag}$ $\left\{Z_{c 1}, \cdots, Z_{c N}\right\}, Z_{c i} \in P^{r_{i} \times p_{i}}, i \in \boldsymbol{N}$ for $Z$ has at least $\eta$ poles in $\mathcal{C}_{+}$with multiplicities.

ii) Let $Z$ satisfy (10). Given any nonnegative integers $n_{i}, i \in \boldsymbol{N}$ where $\Sigma_{i=1}^{N} n_{i}-\eta$ is a nonnegative and even number, there exists a decentralized stabilizing controller $Z_{c}=\operatorname{diag}\left\{Z_{c 1}, \cdots, Z_{c N}\right\}, Z_{c i} \in P^{r_{i} \times p_{i}}, i \in$ $N$ for $Z$ where $Z_{c i}$ has exactly $n_{i}$ poles in $\mathcal{C}_{+}$with multiplicities, $i \in \boldsymbol{N}$.

Proof: i) The proof will be given by induction. We first state the following two results which concern the identification of the (centralized) blocking zeros of $V$ from the system matrix associated with bicoprime fractions.

Let $V \in P^{p \times r}$ and let $V=V_{l} V_{d}^{-1} V_{r}$ be a fractional representation of $V$ over $S$ with $V_{d}$ of size $q \times q$.

Lemma 5: For any $s_{0} \in \mathcal{C}_{+e}$ for which $V\left(s_{0}\right)=0$, one has

$$
\operatorname{rank}\left[\begin{array}{cc}
V_{d} & V_{r} \\
-V_{l} & 0
\end{array}\right]\left(s_{0}\right) \leq q
$$

where equality is achieved if either $\left(V_{d}, V_{l}\right)$ is right coprime or $\left(V_{d}, V_{r}\right)$ is left coprime over $\boldsymbol{S}$.

Lemma 6: If $V=V_{l} V_{d}^{-1} V_{r}$ is a bicoprime fraction over $S$, then for any $s_{0} \in \mathcal{C}_{+e}$

$$
\operatorname{rank}\left[\begin{array}{cc}
V_{d} & V_{r} \\
-V_{l} & 0
\end{array}\right]\left(s_{0}\right)=q
$$

if and only if $V\left(s_{0}\right)=0$.

Now let $N=2$ and note that

$$
\begin{aligned}
& \Psi=\left\{s \in \mathcal{R}_{+e} \mid Z_{11}(s)=0, Z_{21}(s)=0, \text { and } Z_{22}(s)=0\right\} \\
& \cdot \cup\left\{s \in \mathcal{R}_{+e} \mid Z_{22}(s)=0, Z_{12}(s)=0, \text { and } Z_{11}(s)=0\right\} \\
&=\left\{s \in \mathcal{R}_{+e} \mid Z_{11}(s)=0 \text { and } Z_{22}(s)=0\right\} \\
& \cdot \cap\left\{s \in \mathcal{R}_{+e} \mid Z_{21}(s)=0 \text { or } Z_{12}(s)=0\right\} .
\end{aligned}
$$

If $s \in \Psi$ satisfies $\left[Z_{11}^{\prime} Z_{21}^{\prime}\right]^{\prime}(s)=0$, then applying Lemma 5 with $Z:=\left[Z_{11}^{\prime} Z_{21}^{\prime}\right]^{\prime}, P:=\left[P_{1}^{\prime} P_{2}^{\prime}\right]^{\prime}$, and $R:=R_{1}$ we have

$$
\operatorname{rank}\left[\begin{array}{cc}
Q & R_{1} \\
-P_{1} & 0 \\
-P_{2} & 0
\end{array}\right](s)=q
$$

where strict equality holds by the fact that $\left(Q, P_{1}, P_{2}\right)$ is right coprime. If $s \in \Psi$ satisfies $\left[Z_{11} Z_{12}\right](s)=0$, then applying Lemma 5 with $Z:=\left[Z_{11} Z_{12}\right], P:=P_{1}$, and $R:=\left[R_{1} R_{2}\right]$ we have

$$
\operatorname{rank}\left[\begin{array}{ccc}
Q & R_{1} & R_{2} \\
-P_{1} & 0 & 0
\end{array}\right](s)=q
$$

where the strict equality holds since $\left(Q, R_{1}, R_{2}\right)$ is left coprime.
Let $Z_{c i} \in P^{r_{i} \times p_{i}}, i=1,2$ be the transfer matrices of some compensators with the number of unstable poles $n_{1}$ and $n_{2}$, respectively, counted with multiplicities. Also assume that $\operatorname{diag}\left\{Z_{c 1}, Z_{c 2}\right\}$ solves DSP for $Z$. Let $Z_{c 2}=P_{c 2} Q_{c 2}^{-1}$ be a right coprime representation over $S$. Then, Theorem 3.2 of [13] implies that

$$
\hat{Z}\left(Z_{c 2}\right):=\left[P_{1} 0\right]\left[\begin{array}{cc}
Q & R_{2} P_{c 2} \\
-P_{2} & Q_{c 2}
\end{array}\right]^{-1}\left[\begin{array}{c}
R_{1} \\
0
\end{array}\right]
$$

is a bicoprime fraction and $\left(\hat{Z}\left(Z_{c 2}\right), Z_{c 1}\right)$ is stable. For any $s \in \mathcal{R}_{+e}$ for which (12) or (13) holds, it is easy to see that

$$
\operatorname{rank}\left[\begin{array}{ccc}
Q & R_{2} P_{c 2} & R_{1} \\
-P_{2} & Q_{c 2} & 0 \\
-P_{1} & 0 & 0
\end{array}\right](s)=q+p_{2} .
$$

Using the bicoprimeness of the fraction (14) and applying Lemma 6 to $\hat{Z}\left(Z_{c 2}\right)$, we have that every $s \in \Psi$ is an $\mathcal{R}_{+e^{-}}$ blocking zero of $\hat{Z}\left(Z_{c 2}\right)$. From the proof of Theorem 1 in [29] $Z_{c 1}$ stabilizes $\hat{Z}\left(Z_{c 2}\right)$ only if the number of sign changes of

$$
\operatorname{det}\left(\left[\begin{array}{cc}
Q & R_{2} P_{c 2} \\
-P_{2} & Q_{c 2}
\end{array}\right]\right)
$$

in the sequence $\sigma_{1}, \sigma_{2}, \cdots, \sigma_{t}$ is not greater than $n_{1}$, the number of unstable poles $Z_{c 1}$. (Since each $\sigma_{i}$ is an $\mathcal{R}_{+e}$-blocking zero of $\hat{Z}\left(Z_{c 2}\right)$, determinant in (15) is nonzero when evaluated at any $\sigma_{i}$ and therefore its sign in the sequence $\sigma_{1}, \sigma_{2}, \cdots, \sigma_{t}$ is well defined.) On the other hand, for any $s \in \Psi$ it holds that $Z_{22}(s)=0$. Therefore, the number of sign changes of the determinant in (15) and that of $\operatorname{det}(Q) \cdot \operatorname{det}\left(Q_{c 2}\right)$ in the sequence $\sigma_{1}, \sigma_{2}, \cdots, \sigma_{t}$ are equal. It follows that the number of sign changes of $\operatorname{det}(Q)$ in this sequence equals $\eta$ (the number of odd integers in the set $\left.\left\{\eta_{1}, \eta_{2}, \cdots, \eta_{t-1}\right\}\right)$. Then, $\operatorname{det}(Q) \cdot \operatorname{det}\left(Q_{c 2}\right)$ has at least $\eta-n_{2}$ sign changes in the sequence $\sigma_{1}, \sigma_{2}, \cdots, \sigma_{t}$. In other words, for $Z_{c 1}$ to stabilize $\hat{Z}\left(Z_{c 2}\right)$ it must hold that $\eta-n_{2} \leq n_{1}$. This establishes the basis of induction for $N=2$.

Now assume that the statement holds true for $L$. We will establish the statement for $N:=L+1$. Let $Z_{c i}$ with $n_{i}$ unstable poles for $i \in \boldsymbol{N}$ solve DSP for $Z$. Let $Z_{c N}=$ $P_{c N} Q_{c N}^{-1}$ be a right coprime fraction over $S$ of $Z_{c N}$ and consider $\hat{Z}\left(Z_{c N}\right)$ and its induced fraction in (9). By Theorem 3.2 of [10], (9) is a bicoprime fraction and DSP for $\hat{Z}\left(Z_{c N}\right)$ is solvable. Let $\Psi_{L}(\hat{Z})$, namely the set of real unstable decentralized blocking zeros of $Z\left(\hat{Z}_{c N}\right)$, be as defined by (11). By Lemma 3, we have $\Psi \subset \Psi_{L}(\hat{Z})$ and, by Lemma 2 , the elements of $\Psi_{L}(\hat{Z})$ and the poles of $\hat{Z}\left(Z_{c N}\right)$ are disjoint. Let $\bar{\sigma}_{1}, \bar{\sigma}_{2}, \cdots, \bar{\sigma}_{\bar{t}}$ denote the elements of $\Psi_{L}(\hat{Z})$ arranged in the ascending order. Also let $\bar{\eta}_{i}$ denote the number of $\mathcal{R}_{+}$ poles of $\hat{Z}\left(Z_{\mathrm{cN} N}\right)$ counted with multiplicities in the interval $\left(\bar{\sigma}_{i}, \bar{\sigma}_{i+1}\right), i \in\{1,2, \cdots, \bar{t}-1\}$. (Clearly, every unstable pole of $\hat{Z}\left(Z_{c N}\right)$ is an unstable zero of

$$
\operatorname{det}\left(\left[\begin{array}{cc}
Q & R_{N} P_{c N} \\
-P_{N} & Q_{c N}
\end{array}\right]\right)
$$

with the same multiplicity and vice versa.) By the inductive hypothesis, the number of odd integers in the sequence $\bar{\eta}_{1}, \bar{\eta}_{2}, \cdots, \bar{\eta}_{\bar{t}-1}$ is less than or equal to $\Sigma_{i=1}^{L} n_{i}$. In this case 
the number of sign changes of the determinant (16) in the sequence $\sigma_{1}, \sigma_{2}, \cdots, \sigma_{t}$ is not greater than $\Sigma_{i=1}^{L} n_{i}$. Also, in this sequence $(16)$ and $\operatorname{det}(Q) \cdot \operatorname{det}\left(Q_{c N}\right)$ takes the same sign as every decentralized blocking zero $s$ of $Z$ satisfies $Z_{N N}(s)=0$. The number of sign changes of $\operatorname{det}(Q)$. $\operatorname{det}\left(Q_{c N}\right)$ in this sequence is no less than $\eta-n_{N}$, where $\eta$ is the number of sign changes of $\operatorname{det}(Q)$ in $\sigma_{1}, \sigma_{2}, \cdots, \sigma_{t}$, which is precisely the number of odd integers in the set $\left\{\eta_{1}\right.$, $\left.\eta_{2}, \cdots, \eta_{t-1}\right\}$. That is $\eta-n_{N} \leq n_{1}+n_{2}+\cdots+n_{L}$. Since the number of unstable poles of $Z_{c}$ is equal to $\Sigma_{i=1}^{N} n_{i}$, the proof of i) is completed.

ii) For the proof of the second statement we first consider the simplest case where $\Sigma_{i=1}^{N} n_{i}=\eta$. Applying Lemma 4 inductively we obtain compensators $Z_{c N}, \cdots, Z_{c 2}$ with $n_{N}, \cdots, n_{2}$ poles in $\mathcal{C}_{+}$counted with multiplicities, respectively, such that the following fraction of the closed-loop single channel plant is bicoprime

$$
\tilde{Z}:=\left[\begin{array}{c}
P_{1}^{\prime} \\
0 \\
\vdots \\
0
\end{array}\right]^{\prime}\left[\begin{array}{cccc}
Q & R_{N} P_{c N} & \cdots & R_{2} P_{c 2} \\
-P_{N} & Q_{c N} & \cdots & 0 \\
\vdots & \vdots & \ddots & \vdots \\
-P_{2} & 0 & \cdots & Q_{c 2}
\end{array}\right]^{-1}\left[\begin{array}{c}
R_{1} \\
0 \\
\vdots \\
0
\end{array}\right]
$$

and has the following property: If $\tilde{\sigma}_{1}, \tilde{\sigma}_{2}, \cdots, \tilde{\sigma}_{\tilde{t}}$ denote the $\mathcal{R}_{+e}$ blocking zeros of $\tilde{Z}$ arranged in the ascending order and if $\tilde{\eta}_{i}$ denotes the number of $\mathcal{R}_{+}$poles of $\tilde{Z}$ counted with multiplicities in the interval $\left(\tilde{\sigma}_{i}, \tilde{\sigma}_{i+1}\right), i \in\{1,2, \cdots, \tilde{t}-1\}$, it holds that $\tilde{\eta}=\eta-\sum_{i=1}^{L} n_{i}$ where $\tilde{\eta}$ is the number of odd integers in the sequence $\tilde{\eta}_{1}, \cdots, \tilde{\eta}_{\tilde{t}-1}$. Then, $n_{1}-\tilde{\eta}=\mathbf{0}$ and Theorem 1-ii) implies the existence of $Z_{c 1}$ such that $Z_{c 1}$ has $n_{1}$ poles in $\mathcal{C}_{+}$counted with multiplicities and $\left(\tilde{Z}, Z_{c 1}\right)$ is stable. Consequently, $\operatorname{diag}\left\{Z_{c 1}, \cdots, Z_{c N}\right\}$ is a solution to DSP for $Z$. Moreover the compensator $Z_{c i}$ has $n_{i} \mathcal{C}_{+}$poles counted with multiplicities, $i \in N$.

The general case where $\Sigma_{i=1}^{N} n_{i}-\eta$ is a nonnegative even number is treated similarly; however a modification on Lemma 4 is needed. Due to its complex nature, we omit the modified version of Lemma 4 and give only a sketch of the proof for the case $N=2$. The case $N \geq 2$ can be handled similarly.

Let $n_{1}+n_{2}-\eta$ be a nonnegative real number. A local compensator $Z_{c 2}$ around channel 2 can be found such that the induced fraction (14) of $\tilde{Z}=\hat{Z}\left(Z_{c 2}\right)$ is bicoprime and $Z_{c 2}$ has $n_{2}$ poles in $\mathcal{C}_{+}$with multiplicities. These poles are allocated in such a way that $\bar{n}_{2}$ of them are real whereas the others are nonreal where $\bar{n}_{2}$ is the maximum integer for which $\bar{n}_{2} \leq \min \left(\eta, n_{2}\right)$ and $n_{2}-\bar{n}_{2}$ is an even number. Moreover, if $\tilde{\sigma}_{1}, \cdots, \tilde{\sigma}_{\tilde{t}}$ denote the $\mathcal{R}_{+e}$ blocking zeros of $Z$ in the ascending order and if $\tilde{\eta}_{i}$ denotes the number of $\mathcal{R}_{+}$poles of $\tilde{Z}$ counted with multiplicities in the interval $\left(\tilde{\sigma}_{i}, \tilde{\sigma}_{i+1}\right), i \in$ $\{1, \cdots, \tilde{t}-1\}$, it holds that $\tilde{\eta}=\eta-\bar{n}_{2}$ where $\tilde{\eta}$ is the number of odd integers in the sequence $\tilde{\eta}_{i}, i=1, \cdots, \tilde{t}-1$. Observe that if $n_{2} \leq \eta$ then $\bar{n}_{2}=n_{2}$, if $n_{2}>\eta$ and $n_{2}-\eta$ is even then $\bar{n}_{2}=\eta$, and if $n_{2}>\eta$ and $n_{2}-\eta$ is odd then $\bar{n}_{2}=\eta-1$. In all cases $n_{1}+\bar{n}_{2}-\eta=n_{1}-\tilde{\eta}$ is a nonnegative even number as $n_{1}+n_{2}-\eta$ is even. Applying Theorem 1-ii) we obtain a compensator $Z_{\mathrm{c} 1}$ which has $n_{1}$ poles in $\mathcal{C}_{+}$with multiplicities and $\left(\hat{Z}\left(Z_{\mathrm{c} 2}\right), Z_{c 1}\right)$ is stable. This completes the proof.
Remark 4: On comparing Theorems 1 and 2, we conclude that the "least possible" unstable order (McMillan degree) of centralized and decentralized stabilizing controllers are determined, respectively, by the number of odd distributions of $\mathcal{R}_{+}$poles among $\mathcal{R}_{+e}$ blocking zeros of $Z$ and among the $\mathcal{R}_{+e}$ decentralized blocking zeros of $Z$. For those plants satisfying (10), also observe that the unstable poles of the local controllers can be arbitrarily spread among the local controllers. (For the investigation of a similar problem, see [1].)

\section{A. Decentralized Strong Stabilization Problem}

We can now state a solution to DSSP. The result is immediately obtained on noting that $\eta=0$ is a necessary condition for the solvability of DSSP by part i) of Theorem 2 .

Corollary 1: For the $N$-channel plant $Z$, consider the following condition

$$
\begin{aligned}
& Z \text { has no } \mathcal{C}_{+} \text {decentralized fixed modes, and there } \\
& \text { are an even number of real unstable poles of } Z \\
& \text { between each pair of zeros in the set } \Psi .
\end{aligned}
$$

i) DSSP is solvable only if (17) is satisfied.

ii) DSSP is solvable if (10) and (17) are satisfied.

By using various different characterizations of the $\mathcal{R}_{+e}$ decentralized blocking zeros given in Section III, it is possible to obtain many interesting sufficient conditions for the solvability of DSSP under assumption (10). One obvious condition is that $\Psi$ has at most one element since then any set of $\mathcal{R}_{+}$poles will have parity interlacing property with $\Psi$. We state five less obvious conditions below for those plants which have no $\mathcal{C}_{+}$decentralized fixed modes and satisfy (10): Condition a) follows by (6) and b) by the definition of $\mathcal{S}_{Z}$ and by the fact that any symmetric permutation of block rows and columns will include either $Z_{i j}$ or $Z_{j i}$ in its lower triangular for any $i \neq j$. Condition $c$ ) follows by the fact that every decentralized blocking zero of $Z$ is actually a common blocking zero of various complementary transfer matrices. (See Section III.) Condition d) is a consequence of Remark 1. Condition e) can be proven as follows. From Lemma 2, at every $\mathcal{C}_{+e}$ decentralized blocking zero $s_{0}$ of $Z$, it holds that $Z\left(s_{0}\right) \in \mathcal{C}^{p \times r}$ and $\operatorname{rank} Z\left(s_{0}\right)<\min (p, r)$ which imply that $s_{0}$ is a transmission zero of $Z$ [18] provided $Z$ is full rank. Hence, (e) implies that $Z$ has no $\mathcal{R}_{+}$decentralized blocking zeros.

Corollary 2: Let $Z=\left[Z_{i j}\right]$ have no $\mathcal{C}_{+}$decentralized fixed modes and satisfy (10). Then, each of the following conditions implies the solvability of DSSP for $Z$ :

a) There exist $i \in N$ for which $Z_{i i}$ has no $\mathcal{R}_{+}$blocking zeros.

b) There exist $i, j \in N$ with $i \neq j$ for which $Z_{i j}$ and $Z_{j i}$ have no $\mathcal{R}_{+}$blocking zeros.

c) Every complementary transfer matrix of $Z$ is free of $\mathcal{R}_{+}$blocking zeros.

d) There exists $i \in \boldsymbol{N}$ such that the $(q+1)$-st invariant factor of system $\left(P_{i}, Q, R_{i}\right)$ has no $\mathcal{R}_{+}$zeros, i.e., 
equivalently

$$
\operatorname{rank}\left(\left[\begin{array}{cc}
Q & R_{i} \\
-P_{i} & 0
\end{array}\right]\right)(s) \geq q+1, \forall s \in \mathcal{R}_{+} .
$$

e) The plant $Z$ is full rank and has no $\mathcal{R}_{+}$transmission zeros.

The below algorithm summarizes the procedure for obtaining a solution to DSSP for two-channel systems which satisfy (10) and (17). This algorithm can be modified to $N$-channel systems, where $N \geq 3$, for the solution of DSSP and for the construction of decentralized stabilizing controllers with minimum number of unstable poles. (For details, see the proof of Lemma 4 in [27].)

Initiation: Consider a bicoprime fractional representation of $Z$ as

$$
Z=\left[\begin{array}{ll}
Z_{11} & Z_{12} \\
Z_{21} & Z_{22}
\end{array}\right]=\left[\begin{array}{l}
P_{1} \\
P_{2}
\end{array}\right] Q^{-1}\left[\begin{array}{ll}
R_{1} & R_{2}
\end{array}\right] .
$$

Without loss of generality we can assume that $\operatorname{det}(Q)$ takes positive sign at $\sigma_{1}, \sigma_{2}, \cdots, \sigma_{t}$.

Let some left and right coprime fractions of $Z_{22}$ over $S$ be given by $Z_{22}=D_{l}^{-1} N_{l}=N_{r} D_{r}^{-1}$. Let $\Omega_{l}:=\operatorname{gcl} f\left(Q, R_{2}\right)$, so that $Q=\Omega_{l} \tilde{Q}, R_{2}=\Omega_{l} \bar{R}_{2}$, for a left coprime pair of matrices $\left(\tilde{Q}, \bar{R}_{2}\right)$. Also let $\Omega_{r}:=\operatorname{gcr} f\left(\tilde{Q}, P_{2}\right)$ so that $\tilde{Q}$ $=\bar{Q} \Omega_{r}, P_{2}=\bar{P}_{2} \Omega_{r}$, for a right coprime pair of matrices $\left(\bar{Q}, \bar{P}_{2}\right)$. Then, a bicoprime fraction of $Z_{22}$ over $S$ is given by $\bar{P}_{2} \bar{Q}^{-1} \bar{R}_{2}$. Also note that $\operatorname{det}\left(D_{l}\right)=\operatorname{det}\left(D_{r}\right)=\operatorname{det}(\bar{Q})$. Let $\boldsymbol{\Omega}:=\left\{s \in \mathcal{R}_{+e} \mid\left(\operatorname{det}\left(\Omega_{l}\right) \cdot \operatorname{det}\left(\Omega_{r}\right)\right)(s) \neq 0\right\}, D:=$ $\left\{s \in \mathcal{R}_{+e} \mid \operatorname{det}\left(D_{l}\right)(s)=0\right\}, \tilde{\Psi}:=\left\{s \in \mathcal{C}_{+e} \mid\left[Z_{11} Z_{12}\right](s)=\right.$ 0 or $\left.\left[Z_{11}^{\prime} Z_{21}^{\prime}\right]^{\prime}(s)=0\right\}$, and $\bar{\Psi}:=\tilde{\Psi} \cap \mathcal{R}_{+e} . D$ is the set of real unstable poles of $Z_{22}$ and $\Omega$ is the set of extended real numbers excluding the input decoupling and output decoupling zeros of $\left(P_{2}, Q, R_{2}\right)$. Define $\hat{\Psi}=\boldsymbol{\Omega} \cap\{\boldsymbol{D} \cup \bar{\Psi}\}$. It should be noted that $\Psi \subset \hat{\Psi}$. (To see this, let $s \in \Psi$. Then $s$ cannot be a pole of $Z$ via Lemma 2. Consequently, $s \in \boldsymbol{\Omega}$. By definition, $s \in \bar{\Psi}$. Hence, $s \in \hat{\Psi}$.) Let $\Pi:=\hat{\Psi}-\Psi$. It is important to note that for any $s \in \Pi, N_{l}(s) \neq 0$. (This can be proven as follows. $N_{l}(s)=0$ if and only if $Z_{22}(s)=0$, whenever $s \in \mathcal{C}_{+e}$ [29]. This shows that $s$, satisfying $N_{l}(s)=0$, cannot be an element of $D$ and therefore $s$ is an element of $\bar{\Psi}$. This implies $s \in \Psi$. Now, by definition, $s \in \Pi$ implies $N_{l}(s) \neq 0$.)

Step 1:

1) Construct $Z_{c 2} \in S^{r_{2} \times p_{2}}$ using known interpolation techniques and the genericity properties of the ring $S$ to satisfy

a) $\operatorname{det}\left(\Omega_{l}\right) \operatorname{det}\left(\Omega_{r}\right) \operatorname{det}\left(D_{l}+N_{l} Z_{c 2}\right)$ takes nonzero values with positive sign on the elements of $\Pi$. (This can easily be done using the fact that $s \in \Pi \Rightarrow$ $N_{l}(s) \neq 0$. Also see [24, Theorem 2.2]).

b) The pairs $\left(D_{l}, Z_{c 2}\right)$ and $\left(D_{r}, Z_{c 2}\right)$ are right and left coprime, respectively.

c) The following fractional representation is bicoprime over $S$

$$
\hat{Z}\left(Z_{c 2}\right)=\left[\begin{array}{ll}
P_{1} & 0
\end{array}\right]\left[\begin{array}{cc}
Q & R_{2} Z_{c 2} \\
-P_{2} & I
\end{array}\right]^{-1}\left[\begin{array}{c}
R_{1} \\
0
\end{array}\right] .
$$

Property a) yields that $\operatorname{det}\left(\Omega_{l}\right) \operatorname{det}\left(\Omega_{r}\right) \operatorname{det}\left(D_{l}+N_{l}\right.$ $Z_{c 2}$ ) takes nonzero values with positive sign on the elements of $\hat{\Psi}$. (Recall that $\Pi=\hat{\Psi}-\Psi$ and $\operatorname{det}\left(\Omega_{l}\right)$ $\operatorname{det}\left(\Omega_{r}\right) \operatorname{det}\left(D_{l}\right)=\operatorname{det}(Q)$ takes positive sign at the elements of $\Psi$.) Property b) is employed in Step 2 below. Property c) means that the resulting single channel system around channel 1 resulting by the application of $Z_{c 2}$ around channel 2 is stabilizable and detectable.

2) If all the $\mathcal{R}_{+e}$ blocking zeros of $\hat{Z}\left(Z_{c 2}\right)$ are contained in $\hat{\Psi}$, then let $\Delta_{c}=0_{r_{2} \times p_{2}}$ and go to Step 3. Otherwise, go to Step 2.

Step 2: First note that there exists $\delta_{1}>0$ such that a), b), c) of Step 1-1) are still satisfied when $Z_{c 2}$ is replaced by $Z_{c 2}+\Delta$, for every $\Delta \in S^{r_{2} \times p_{2}}$ satisfying $\|\Delta\|<\delta_{1}$.

1) Let $T:=Z_{c 2}\left(I+Z_{22} Z_{c 2}\right)^{-1}$ and let $T_{1}^{-1} T_{2}=T$ be a left coprime fraction of $T$ over $S$. It holds that $Z_{c 2}=D_{r}\left(T_{1} D_{r}-T_{2} N_{r}\right)^{-1} T_{2}$. Since $\left(D_{r}, Z_{c 2}\right)$ is left coprime, $\left(T_{1} D_{r}-T_{2} N_{r}\right) D_{r}^{-1}$ is over $S$, i.e., $T_{2}=\tilde{T}_{2} D_{l}$ for some matrix $\tilde{T}_{2}$ over $S$. Let $T_{1}^{-1} \tilde{T}_{2}=\hat{T}_{2} \hat{T}_{1}^{-1}$, for a right coprime pair of matrices $\left(\hat{T}_{2}, \hat{T}_{1}\right)$. It follows that $Z_{c 2}=\hat{T}_{2}\left(\hat{T}_{1}-N_{l} \hat{T}_{2}\right)^{-1} D_{l}$. By the right coprimeness of $\left(\hat{T}_{1}-N_{l} \hat{T}_{2}, \hat{T}_{2}\right)$ and by the right coprimeness of $\left(D_{l}, Z_{c 2}\right)$, it easily follows that $D_{l}=\left(\hat{T}_{1}-N_{l} \hat{T}_{2}\right) V$ for some unimodular $V$ over $S$ and $Z_{c 2}=\hat{T}_{2} V$. For $\delta_{2}:=1 /\left\|V N_{r}\right\|, V^{-1}-N_{r} \Delta$ is unimodular for every $\Delta \in S^{r_{2} \times p_{2}}$ satisfying $\|\Delta\|<\delta_{2}$.

2) There exists an open and dense subset $\mathcal{X}$ of $\boldsymbol{S}^{\boldsymbol{r}_{2} \times p_{2}}$ such that for any fixed but otherwise arbitrary $\Delta \in \mathcal{X}$, the implication

$$
\begin{gathered}
\left(Z_{11}-Z_{12}\left(\hat{T}_{2}+D_{r} \Delta\right) \hat{T}_{1}^{-1} D_{l} Z_{21}\right)(s)=0 \\
\Rightarrow\left[Z_{11} Z_{12}\right](s)=0 \text { or }\left[Z_{11}^{\prime} Z_{21}^{\prime}\right]^{\prime}(s)=0 \\
\forall s \in \mathcal{R}_{+e}-\boldsymbol{D}
\end{gathered}
$$

holds. (See [27] and [22].) Let $\Delta \in \mathcal{X}$ be such that $\|\Delta\|<\delta_{2}$ and $\left\|Z_{c 2}-Z_{\mathrm{c} 2 \Delta}\right\|<\delta_{1}$ where $Z_{\mathrm{c} 2 \Delta}:=\left(\hat{T}_{2}+\right.$ $\left.D_{r} \Delta\right)\left(V^{-1}-\hat{N}_{r} \Delta\right)^{-1}$. Now, $Z_{c 2 \Delta}$ belongs to $S^{r_{2} \times p_{2}}$ and $\mathrm{a}), \mathrm{b}), \mathrm{c}$ ) of Step 1-1 are all satisfied when $Z_{c 2}$ is replaced by $Z_{c 2 \Delta}$. Define $\Delta_{c}=Z_{c 2 \Delta}-Z_{c 2}$. By Step 1-1.c), the set of $\mathcal{R}_{+e}$ blocking zeros of $\hat{Z}\left(Z_{c 2}+\Delta_{c}\right)$ is disjoint from the set of $\mathcal{R}_{+e}$ zeros of $\operatorname{det}\left(\Omega_{l}\right) \operatorname{det}\left(\Omega_{r}\right)$, i.e., the set of $\mathcal{R}_{+e}$ blocking zeros of $\hat{Z}\left(Z_{c 2}+\Delta_{c}\right)$ is included in $\boldsymbol{\Omega}$. Implication (18) now shows that the $\mathcal{R}_{+e}$ blocking zeros of $\hat{Z}\left(Z_{c 2}+\Delta_{c}\right)$ are all contained in $\hat{\Psi}$. This implies via Step 1-1.a) that there exists an even number of real unstable poles of $\hat{Z}\left(Z_{c 2}+\Delta_{c}\right)$ between each pair of real unstable blocking zeros.

Step 3: Using known techniques determine $Z_{c 1} \in S^{r_{1} \times p_{1}}$ such that $\left(\hat{Z}\left(Z_{c 2}+\Delta_{c}\right), Z_{c 1}\right)$ is stable. It is satisfied via c) of Step 1 that $\left(Z, \operatorname{diag}\left\{Z_{c 1}, Z_{c 2}+\Delta_{c}\right\}\right)$ is stable. This terminates the algorithm.

Remark 5: In the above algorithm, the fundamental step is Step 1 where a) and c) ensure that there are an even number of real unstable poles of $\hat{Z}\left(Z_{c 2}\right)$ between each pair of elements in the set $\hat{\Psi}$. Statement $b$ ) is of technical importance only and is utilized in Step 2. Note that for any $Z_{c 2}$ satisfying 1-1.c), the set of $\mathcal{R}_{+e}$ blocking zeros of $\hat{Z}\left(Z_{c 2}\right)$ contains $\Psi$ (Lemma 3 ) and $\Psi \subset \hat{\Psi}$. In Step 2 , we perturb $Z_{c 2}$ slightly to $Z_{c 2}+\Delta_{c}$ to further satisfy that the $\mathcal{R}_{+e}$ blocking zeros of $\hat{Z}\left(Z_{c 2}+\Delta_{c}\right)$ are 
confined to $\hat{\Psi}$. This completes the design of the second channel local controller and reduces the problem to the (centralized) strong stabilization problem around channel 1 . This problem is solved via known methods at Step 3.

What makes Step 2 so complicated is that the perturbation on $Z_{c 2}$ should be "directional," although the norm of the perturbation matrix $\Delta_{c}$ can always be chosen arbitrarily small. Implication (18) in Step 2 is valid only if A2) of (10) is satisfied. (See also Section IV-B.)

The complement of $\Psi$ in $\hat{\Psi}$ consists of those $\mathcal{R}_{+}$poles of $Z_{22}$ which are not input decoupling or output decoupling zeros of $\left(P_{2}, Q, R_{2}\right)$. If the above algorithm is modified for the construction of decentralized stabilizing controllers with minimum number of unstable poles, the set $\Psi$ will also include the unstable poles of $Z_{c 2}$. (See [27].)

In various examples, we have observed that at the end of Step 1 the set of $\mathcal{R}_{+e}$ blocking zeros of $\hat{Z}\left(Z_{c 2}\right)$ is already contained in $\hat{\Psi}$. In such cases we do not need to find out a perturbation matrix so that Step 2 can be skipped.

An example is given below to demonstrate the algorithm.

Example 1: Let $Z$ below be the transfer matrix of a twochannel system as shown in the equation at the bottom of the page where $Z_{11} \in \boldsymbol{P}^{2 \times 1}, Z_{12} \in \boldsymbol{P}^{2 \times 2}, Z_{21} \in \boldsymbol{P}$ and $Z_{22} \in \boldsymbol{P}^{1 \times 2}$. The plant $Z$ has no $\mathcal{C}_{+}$decentralized fixed modes and $\Psi=\{\infty\}$. That is, $Z$ is decentralized strong stabilizable.

Initiation: A bicoprime fraction of $Z$ over $\boldsymbol{S}$ is given by $\left[P_{1}^{\prime} P_{2}^{\prime}\right]^{\prime} Q^{-1}\left[R_{1} R_{2}\right]$ where

$$
\begin{aligned}
& P_{1}=\left[\begin{array}{ccc}
\frac{(s-1)}{(s+1)^{2}} & 0 & 0 \\
0 & \frac{1}{(s+1)} & \frac{1}{(s+1)}
\end{array}\right], \\
& P_{2}=\left[\begin{array}{ccc}
\frac{1}{(s+1)} & \frac{1}{(s+1)} & 0
\end{array}\right], \\
& R_{1}=\left[\begin{array}{c}
\frac{1}{(s+1)} \\
\frac{1}{(s+1)} \\
\frac{1}{(s+1)}
\end{array}\right], \quad R_{2}=\left[\begin{array}{cc}
\frac{(s-1)}{(s+1)^{2}} & \frac{1}{(s+1)} \\
\frac{1}{(s+1)} & \frac{1}{(s+1)}
\end{array}\right]
\end{aligned}
$$

and

$Q=\operatorname{diag}\{(s-1) /(s+1),(s-2) /(s+1),(s-3) /(s+1)\}$.

One obtains

$$
\begin{aligned}
& D_{l}=\frac{(s-1)(s-2)}{(s+1)^{2}}, \quad N_{l}=\left[\frac{(s-1)(2 s-1)}{(s+1)^{4}} \frac{(2 s-3)}{(s+1)^{3}}\right], \\
& D_{r}=\left[\begin{array}{ll}
\frac{-3(s-1)}{(s+1)} & \frac{(s-1)(s-2)}{(s+1)^{2}}
\end{array}\right],
\end{aligned}
$$

$$
\begin{aligned}
N_{r} & =\left[\begin{array}{ll}
\frac{-4}{(s+1)^{2}} & \frac{(2 s-3)}{(s+1)^{3}}
\end{array}\right], \\
\Omega_{l} & =\operatorname{diag}\left\{1,1, \quad \frac{(s-3)}{(s+1)}\right\}, \\
\tilde{Q} & =\operatorname{diag}\left\{\frac{(s-1)}{(s+1)}, \quad \frac{(s-2)}{(s+1)}, 1\right\}, \quad \bar{R}_{2}=R_{2}, \\
\Omega_{r} & =I_{3}, \quad \bar{Q}=\tilde{Q}, \quad \bar{P}_{2}=P_{2}, \\
\boldsymbol{\Omega} & =\mathcal{R}_{+e}-\{3\}, \quad \boldsymbol{D}=\{1,2\}, \\
\bar{\Psi} & =\{\infty\}, \quad \hat{\Psi}=\{1,2, \infty\}, \quad \Psi=\{\infty\}, \quad \Pi=\{1,2\} .
\end{aligned}
$$

Step 1:

1) We let

$$
Z_{c 2}=\left[\begin{array}{ll}
0 & \frac{(-s+3 / 2)}{(s+1)}
\end{array}\right]^{\prime}
$$

which satisfies a), b), and c).

2) It holds that

$$
\hat{Z}\left(Z_{c 2}\right)=\left[\begin{array}{c}
\frac{2(s-1)(s-2)}{\left(2 s^{4}-2 s^{3}-10 s^{2}+14 s-5\right)} \\
\frac{\left(4 s^{4}-6 s^{3}-18 s^{2}+18 s+1\right)}{(s+1)\left(2 s^{4}-2 s^{3}-10 s^{2}+14 s-5\right)}
\end{array}\right] .
$$

$\hat{Z}\left(Z_{c 2}\right)$ has only one $\mathcal{R}_{+e}$ blocking zero, $\infty$, which is contained in $\hat{\Psi}$. We therefore go directly to Step 3 with $\Delta=0_{2 \times 1}$.

Step 3: $\hat{Z}\left(Z_{\mathrm{c} 2}\right)$ is strong stabilizable. Using standard results [29] one can construct $Z_{c 1} \in S^{1 \times 2}$ satisfying that $\left(\hat{Z}\left(Z_{c 2}\right), Z_{c 1}\right)$ is stable.

Remark 6: The solvability of DSP together with the strong centralized stabilizability is in general not enough for the solvability of DSSP. This is illustrated by the following example. Let a $2 \times 2$ transfer matrix be given by $Z=\left[Z_{i j}\right]$ where

$$
\begin{aligned}
& Z_{11}=\frac{(s-1)(s-3)}{(s+1)(s-2)(s-4)}, \\
& Z_{12}=\frac{(s-1)(s-3)}{(s+1)(s-2)(s-4)^{2}}, \\
& Z_{21}=\frac{1}{(s+1)}, \quad Z_{22}=\frac{(s-1)(s-3)}{(s+1)^{3}} .
\end{aligned}
$$

It is easily checked that $Z$ has no $\mathcal{C}_{+}$decentralized fixed modes [16]. We have $\Psi=\{1,3, \infty\}, \eta_{1}=1$ (corresponding to the pole at $s=2$ ) and $\eta_{2}=1$ (corresponding to the pole at $s=4$ ). Theorem 2 and Corollary 1 yield that $Z$ is not decentralized strong stabilizable and that any decentralized stabilizing controller of $Z$ has at least $\eta_{1}+\eta_{2}=2$ unstable

$$
Z=\left[\begin{array}{c|cc}
\frac{1}{(s+1)^{2}} & \frac{(s-1}{(s+1)^{3}} & \frac{1}{(s+1)^{2}} \\
\frac{(2 s-5)}{(s-1)(s-2)(s-3)} & \frac{1}{(s-2)(s+1)} & \frac{1}{(s-2)(s+1)} \\
\hline \frac{(2 s-3)}{(s-1)(s+1)(s-2)} & \frac{(2 s-1)}{(s+1)^{2}(s-2)} & \frac{(2 s-2)}{(s+1)(s-1)(s-2)}
\end{array}\right]
$$


poles with multiplicities. On the other hand, since $Z$ has no $\mathcal{R}_{+e}$-blocking zeros except $s=\infty$, it is (centralized) strong stabilizable.

It is known that strong stabilization problem is generically solvable for nonscalar systems [30]. An analogous result for decentralized strong stabilization problem can be given as follows.

Let $\overline{\boldsymbol{P}}^{p \times r}$ be a subset of $\boldsymbol{P}^{p \times r}$ such that $Z \in \overline{\boldsymbol{P}}^{p \times r}$ if and only if (10) hold for $Z$ and $Z$ is devoid of unstable decentralized fixed modes.

Theorem 3: For almost all $Z \in \overline{\boldsymbol{P}}^{p \times r}$, DSSP is solvable, where the quantifier "almost all" is with respect to the subspace topology induced by the graph topology.

A proof of the above theorem is presented in [22] along the same lines as the proof of [29, Theorem 5.3.1]. Its outline can be given as follows. First note that for a plant $Z$ being a member of $\overline{\boldsymbol{P}}^{p \times r}$ is an open property with respect to the subspace topology in $\boldsymbol{P}^{p \times r}$, induced by graph topology. If DSSP is solvable for $Z \in P^{p \times r}$ then there exists a stable decentralized controller which stabilizes all the plants contained in a sufficiently small neighborhood around $Z$ in $P^{p \times r}$ and, consequently, in a sufficiently small neighborhood in $\overline{\boldsymbol{P}}^{p \times r}$. This proves that the set of plants for which DSSP is solvable is open in $\overline{\boldsymbol{P}}^{p \times r}$. In [22], it is also shown that if DSSP for $Z \in \boldsymbol{P}^{p \times r}$ is solvable then $Z$ can be perturbed by an arbitrarily "small" perturbation matrix $\Delta_{Z}$, i.e., $Z \rightarrow Z+\Delta_{Z}$, such that

a) A1) and A2) hold for $Z+\Delta_{Z}$ and $Z+\Delta_{Z}$ is free of unstable decentralized fixed modes, i.e., $Z+\Delta_{Z}$ belongs to $\overline{\boldsymbol{P}}^{p \times r}$ [23], and

b) $\left(Z+\Delta_{Z}\right)_{i_{0} j_{0}}$ and $\left(Z+\Delta_{Z}\right)_{j_{0} i_{0}}$ each has at most one $\mathcal{R}_{+e}$ decentralized blocking zero for some $i_{0}, j_{0} \in$ $N, i_{0} \neq j_{0}$. From Corollary 2b), DSSP for $Z+\Delta_{Z}$ is solvable. This shows that the set of $Z$ for which DSSP is solvable is dense in $\bar{P}^{p \times r}$.

Remark 7: A consequence of the above theorem is that the singular conditions under which the solution of DSSP fails for a plant in $\overline{\boldsymbol{P}}^{p \times r}$ are nongeneric conditions [29, Chap. 7] which can be removed by arbitrarily small perturbations on $Z$ (see also the below subsection). Another consequence is the following: If a decentralized controller $Z_{c}$ solves DSSP for $Z \in \overline{\boldsymbol{P}}^{p \times r}$ then, under sufficiently small modeling errors on $Z$, i) DSSP for $Z$ remains solvable and ii) the stable decentralized controller solving DSSP for $Z$ continues to stabilize $Z$. $\Delta$

\section{B. On Assumptions A1) and A2)}

In Theorem 2, part i) is independent of Assumptions Al) and A2). In part ii), A1) can be assumed to hold without loss of generality. A2) is generically satisfied for a class of multichannel systems. Let a collection of positive integers $p_{i}, r_{i} i \in N$ satisfy that $\left(p_{i} \geq 2\right.$ and $\left.r_{j} \geq 2\right)$ or $\left(p_{j} \geq 2\right.$ and $\left.r_{i} \geq 2\right), \forall i, j \in N, i \neq j$. Define $p=\Sigma_{i=1}^{N} p_{i}, r=\Sigma_{i=1}^{N} r_{i}$. It is straightforward to show that the set of plants satisfying (10) is open and dense in $\boldsymbol{P}^{p \times r}$ with respect to the subspace topology induced by graph topology [22]. A2), however, still excludes some important cases such as a $2 \times 2$ plant. We will examine below why a failure of Assumption A2) complicates the solution of DSSP for $2 \times 2$ plants.

Consider a $2 \times 2$ plant which has no $\mathcal{C}_{+}$decentralized fixed modes: $Z=\left[Z_{i j}\right], Z_{i j} \in \boldsymbol{P}, i=1,2$. Assume $Z$ has a bicoprime representation as in (4) with $N=2$ and $r_{1}=r_{2}$ $=p_{1}=p_{2}=1$. Let

$$
\begin{aligned}
a & :=\operatorname{det}(Q) \quad \text { and } b:=\operatorname{det}\left(\left[\begin{array}{cc}
Q & R_{2} \\
-P_{2} & 0
\end{array}\right]\right), \\
c & :=\operatorname{det}\left(\left[\begin{array}{cc}
Q & R_{1} \\
-P_{1} & 0
\end{array}\right]\right) \\
d & :=\operatorname{det}\left(\left[\begin{array}{ccc}
Q & R_{1} & R_{2} \\
-P_{1} & 0 & 0 \\
-P_{2} & 0 & 0
\end{array}\right]\right) .
\end{aligned}
$$

Let $Z_{c 2} \in S$ be a compensator around the second channel of $Z$ such that the resulting single channel system is stabilizable and detectable from the first channel. Writing an explicit expression for $\hat{Z}\left(Z_{c 2}\right)$ and using Lemmas 5 and 6 it is not difficult to show that the set of $\mathcal{C}_{+e}$ zeros of $\hat{Z}\left(Z_{c 2}\right)$ is precisely the set of $\mathcal{C}_{+e}$ zeros of $c+d Z_{\mathrm{c} 2}$ and the set of $\mathcal{C}_{+}$poles of $\hat{Z}\left(Z_{c 2}\right)$ is precisely the set of $\mathcal{C}_{+}$zeros of $a+b Z_{c 2}$. Solving DSSP for a $2 \times 2$ plant amounts to determining a $Z_{c 2} \in S$ for which the following property holds:

$\mathcal{P}$ : The pair $\left(a+b Z_{c 2}, c+d Z_{c 2}\right)$ is coprime over $S$, the function $a+b Z_{c 2}$ is nonzero and takes the same sign at all $\mathcal{R}_{+e}$ zeros of $c+d Z_{c 2}$.

Let $e$ be a greatest common factor of $c$ and $d$ over $S$ such that $c=\bar{c} e, d=\bar{d} e$. Define

$$
\begin{aligned}
\mathcal{E}:= & \left\{s \in \mathcal{R}_{+e} \mid e(s)=0\right\}, \\
I_{+}:= & \left\{s \in \mathcal{R}_{+e} \mid s \text { is not a zero of } \bar{d}\right. \text { and } \\
& \left.\cdot\left(a-b \frac{\bar{c}}{\bar{d}}\right)(s)>0\right\}, \\
I_{-}:= & \left\{s \in \mathcal{R}_{+e} \mid s \text { is not a zero of } \bar{d}\right. \text { and } \\
& \left.\cdot\left(a-b \frac{\bar{c}}{\bar{d}}\right)(s)<0\right\} .
\end{aligned}
$$

Consider the following lemma whose proof can be found in [27].

Lemma 7: For some $Z_{c 2} \in S$ property $\mathcal{P}$ is satisfied if and only if any of a) or b) holds:

a) i) All the $\mathcal{R}_{+e}$ zeros of $\bar{c}+\bar{d} Z_{\mathrm{c} 2}$ (if any) are contained in $I_{+}$and

ii) (In case $\mathcal{E} \neq \emptyset)\left(a+b Z_{c 2}\right)(s)>0$ at every $s \in \mathcal{E}$.

b) i) All the $\mathcal{R}_{+e}$ zeros of $\bar{c}+\bar{d} Z_{c 2}$ (if any) are contained in $I_{-}$and

ii) (In case $\mathcal{E} \neq \emptyset)\left(a+b Z_{c 2}\right)(s)<0$ at every $s \in \mathcal{E}$.

Let $\mathcal{C}_{-}$denote the open left-half plane and define $\dot{\mathcal{C}}_{+e}=$ $\mathcal{C}_{+e}-\mathcal{R}_{+e}$. The subproblem corresponding to a-i) is that of determining a stable controller $Z_{c 2}$ which places all zeros of $\bar{c}+\bar{d} Z_{c 2}$ in $\mathcal{C}_{-} \cup \dot{\mathcal{C}}_{+e} \cup I_{+}$. Similarly, in b-i) we seek a stable controller $Z_{c 2}$ which places all zeros of $\bar{c}+\bar{d} Z_{\mathrm{c} 2}$ in $\mathcal{C}_{-} \cup \dot{\mathcal{C}}_{+e} \cup I_{-}$. Let, for simplicity, $\bar{c}$ be nonzero. If $\bar{c}^{-1} \bar{d}$ is strongly stabilizable then there always exists a stable $Z_{c 2}$ 
satisfying a-i) or b-ii) but the converse may not be true in general. The subproblems corresponding to a-ii) and $b$-ii) impose extra interpolation constraints on the stable controller. This problem, in its generality, is very complex, and the concept of decentralized blocking zeros is not sufficient to capture its solution alone. Nevertheless, some connections between Corollary 1 and Lemma 7 can be made. For example, the necessity of the parity interlacing property between the set of real unstable poles and real unstable decentralized blocking zeros for the solvability of DSSP is implicit in Lemma 7. In fact, every $\mathcal{C}_{+e}$ decentralized blocking zero of $Z$ is a common zero of $b, c$, and $d$, and $s \in \mathcal{C}_{+}$is a pole of $Z$ if and only if $s$ is a zero of $a$. The necessity of the parity interlacing property now can be deduced from a-ii) and b-ii).

Lemma 7 is also useful in showing that, in general, the existence of the parity interlacing property between the real unstable poles and real unstable decentralized blocking zeros of a $2 \times 2$ plant is not sufficient for the solvability of DSSP. To see this consider the following plant where, for convenience, $P_{1}=\left[\begin{array}{ll}1 & 0\end{array}\right], P_{2}=\left[\begin{array}{ll}0 & 1\end{array}\right]$ (see (19) at the bottom of the page) where

$$
\begin{aligned}
& a=c=\frac{(s-3)(s-5)}{(s+1)^{2}}, \\
& b=\frac{(s-2)(s-4)\left((s+1)^{4}+(s-2)(s-3)(s-4)(s-5)\right)}{(s+1)^{7}}, \\
& d=\frac{(s-2)(s-4)}{(s+1)^{3}}, \quad \bar{c} \\
& a-b \frac{\bar{c}}{\bar{d}}=-\frac{(s-2)(s-3)^{2}(s-4)(s-5)^{2}}{(s+1)^{6}}, \\
& e=1, \quad \bar{c}=c, \quad \bar{d}=d, \quad \mathcal{E}=\emptyset, \\
& I_{-}=[0,2) \cup(4,5) \cup(5, \infty), \quad I_{+}=(2,3) \cup(3,4) .
\end{aligned}
$$

Despite the fact that $Z$ has no decentralized blocking zeros, DSSP for $Z$ is not solvable. This can be shown as follows. For any $Z_{c 2} \in S,\left(c+d Z_{c 2}\right)(2)>0$ and $\left(c+d Z_{c 2}\right)(4)<0$, i.e., $c+d Z_{c 2}$ has a zero in $[2,4]$. It is not possible that all the real unstable zeros of $c+d Z_{c 2}$ are contained in $I_{-}$. Also, for any $Z_{c 2} \in S,\left(c+d Z_{c 2}\right)(\infty)>0$, i.e., $c+d Z_{c 2}$ has a zero in $[4, \infty)$. All the real unstable zeros of $c+d Z_{c 2}$ cannot be contained in $I_{+}$as well.

Even for $2 \times 2$ plants, the solvability of DSSP bears upon a more general concept than the parity interlacing property between the set of real unstable poles and real unstable decentralized blocking zeros. For multivariable, multichannel systems where A2) fails, we anticipate that the solvability pertains to more complicated conditions.

\section{Decentralized ConcurRent Stabilization Problem}

As an application of DSSP, our objective in this section is to define a special decentralized simultaneous stabilization problem, called "decentralized concurrent stabilization problem (DCSP)," and to investigate its applications in the design of local controllers for large-scale systems. In this context, two problems will be considered.

Stabilization of Interconnected Systems Using Locally Stabilizing Subsystem Controllers: Consider a collection of linear time-invariant finite-dimensional systems described by

$$
\Sigma_{i}: \dot{x}_{i}=A_{i} x_{i}+B_{i} v_{i}+u_{i}, \quad y_{i}=C_{i} x_{i}
$$

$i \in \boldsymbol{N}$ where $A_{i} \in \mathcal{R}^{n_{i} \times n_{i}}, B_{i} \in \mathcal{R}^{n_{i} \times r_{i}}$ and $C_{i} \in \mathcal{R}^{p_{i} \times n_{i}}$ correspond to states, inputs and outputs, respectively. Assume that these systems are interconnected according to the rule $u_{i}=\sum_{j=1}^{N} A_{i j} x_{j}, i \in N$ for some $A_{i j} \in \mathcal{R}^{n_{i} \times n_{j}}$. Then, the composite (interconnected) system can be described as $\Sigma: \dot{x}=A x+B v, y=C x$ where $x:=\left[x_{1}^{\prime} \cdots x_{N}^{\prime}\right]^{\prime}$, $y:=\left[y_{1}^{\prime} \cdots y_{N}^{\prime}\right]^{\prime}, v:=\left[v_{1}^{\prime} \cdots v_{N}^{\prime}\right]^{\prime}, B:=\operatorname{diag}\left\{B_{1}, \cdots, B_{N}\right\}$, $C:=\operatorname{diag}\left\{C_{1}, \cdots, C_{N}\right\} . A$ is a matrix consisting of $N$ block rows and columns such that its $(i, j)$ th submatrix is $A_{i j}$, if $i \neq j$, and $A_{i}+A_{i i}$ otherwise. It is assumed that the subsystems $\Sigma_{i}=\left(C_{i}, A_{i}, B_{i}\right), i \in N$ and the composite system $\Sigma=(C, A, B)$ are stabilizable and detectable.

P1) Determine local controllers $\Sigma_{c i}, i \in N$ around subsystems $\Sigma_{i}$ such that

i) the subsystems $\left(\Sigma_{i}, \Sigma_{c i}\right), i \in \boldsymbol{N}$ are all stable and

ii) when the interconnections $A_{i j}$ exist the composite closed-loop system becomes stable, i.e., $\left(\Sigma, \operatorname{diag}\left\{\Sigma_{1}, \cdots, \Sigma_{N}\right\}\right)$ is stable.

Such an approach to the stabilization problem of composite systems is a natural one because most of the composite systems are constructed by interconnecting a finite number of independently controlled subsystems [19]. Therefore, P1) and its variations have attracted considerable attention in control theory. Although there are numerous interesting results on many variations of this problem (see the references in [20, Chap. 3]), a necessary and sufficient solvability condition for the problem is still missing.

$$
\begin{aligned}
Z & =\left[\begin{array}{cc}
1 & \frac{(s-2)(s-4)}{(s+1)^{3}} \\
\frac{(s-2)(s-4)}{(s+1)^{2}} & \frac{(s-2)(s-4)\left(2 s^{4}-10 s^{3}+77 s^{2}-150 s+121\right)}{(s+1)^{5}(s-3)(s-5)}
\end{array}\right] \\
& =\left[\begin{array}{ll}
-\frac{(s-2)(s-3)(s-4)(s-5)}{(s+1)^{4}} & \frac{(s-3)(s-5)}{(s+1)^{2}}
\end{array}\right]^{-1}\left[\begin{array}{cc}
1 & \frac{(s-2)(s-4)}{(s+1)^{3}} \\
0 & \frac{(s-2)(s-4)}{(s+1)^{3}}
\end{array}\right]
\end{aligned}
$$


Stabilization of a Multichannel System via Stabilization of its Main Diagonal Subsystems: Let $Z=\left[Z_{i j}\right], Z_{i j} \in$ $P^{p_{i} \times r_{j}} i, j \in \boldsymbol{N}$ be the transfer matrix of a given plant.

P2) Determine local controllers $Z_{c i}, i \in N$ such that

i) $\left(Z_{i i}, Z_{c i}\right), i \in \boldsymbol{N}$ are all stable and

ii) when the interconnections $Z_{i j}, Z_{i j}, i \neq j$ exist the composite closed-loop system becomes stable, i.e., $\left(Z, \operatorname{diag}\left\{Z_{c 1}, \cdots, Z_{c N}\right\}\right)$ is stable.

A basic difference between P1) and P2) is that, in P1) the subsystems are defined with respect to their state-space realizations whereas in $\mathrm{P} 2$ ) the subsystems are defined with respect to the main diagonal transfer matrices of the interconnected system. Moreover, in P1) the subsystems are feedback interconnected whereas in P2) the subsystems are feedforward interconnected (see [24]). P2) originates from the work of Rosenbrock [17] where the objective is to develop a methodology to extend the well-known SISO frequency domain design techniques to multiloop systems. In a general set-up the plant transfer matrix $Z$ is possibly obtained via a cascade connection of the original plant with some pre- and post-compensators. P2) has found applications especially in the process control problems. A review of the existing results for its solution can be found in [12, Chap. 14]. For related studies, see also [33] and [14]. As in P1), a complete solution procedure for $\mathrm{P} 2$ ) is missing.

We will formulate these problems as a special decentralized simultaneous stabilization problem and obtain their solutions via transforming them to DSSP on suitable auxiliary plants. For the class of plants satisfying A2), this procedure yields complete solvability conditions. For other classes of plants our conditions will serve as necessary conditions for the solvability. The problems P1), P2) can be unified by the following problem of decentralized simultaneous stabilization [22].

Decentralized Concurrent Stabilization Problem (DCSP): Let $Z=\left[Z_{i j}\right], Z_{i j} \in \boldsymbol{P}^{p_{i} \times r_{j}}, i, j \in \boldsymbol{N}$ be the transfer matrix of a given plant where $p:=\Sigma_{i=1}^{N} p_{i}, r:=\Sigma_{i=1}^{N} r_{i}$. Also let $T_{i} \in \boldsymbol{P}^{p_{i} \times r_{i}}, i \in \boldsymbol{N}$ be given. Determine local controllers $Z_{c i} \in P^{r_{i} \times p_{i}}, i \in N$ such that the pairs $\left(T_{i}, Z_{c i}\right), i \in N$ are stable and the pair $\left(Z, \operatorname{diag}\left\{Z_{c 1}, \cdots, Z_{c N}\right\}\right)$ is stable.

Since one of the plants in its definition is block diagonal, DCSP is only a special case of the general problem of simultaneous stabilization of two plants via a decentralized controller. The following theorems can be proven easily using the problem definitions.

Theorem 4: Problem P1) is solvable if and only if DCSP for $Z=C(s I-A)^{-1} B$ and $T_{i}=C_{i}\left(s I-A_{i}\right)^{-1} B_{i}, i \in N$ is solvable.

Theorem 5: Problem P2) is solvable if and only if DCSP for $Z$ and $T_{i}=Z_{i i}, i \in N$ is solvable.

In case of centralized feedback systems, simultaneous stabilization problem can be formulated as a strong stabilization problem [29, Chap. 5.4]. Analogously, the solution of DCSP is obtained by transforming it to a decentralized strong stabilization problem. To do this, we first obtain a suitable auxiliary plant.

Let some left and right coprime fractions of $T_{i}, i \in N$ over $S$ be given as $T_{i}=D_{l i}^{-1} N_{l i}=N_{r i} D_{r i}^{-1}, i \in N$. There exist matrices $K_{i} \in S^{p_{i} \times p_{i}}, L_{i} \in S^{r_{i} \times p_{i}}, \bar{K}_{i} \in S^{r_{i} \times r_{i}}, \bar{L}_{i} \in$ $\boldsymbol{S}^{r_{i} \times p_{i}}, i \in \boldsymbol{N}$ such that

$$
\left[\begin{array}{cc}
D_{l i} & N_{l i} \\
\bar{L}_{i} & -\bar{K}_{i}
\end{array}\right]\left[\begin{array}{cc}
K_{i} & N_{r_{i}} \\
L_{i} & -D_{r_{i}}
\end{array}\right]=I, L_{i}, \bar{L}_{i}
$$

are strictly proper, $i \in \boldsymbol{N}$. Then, $\left(T_{i}, Z_{c i}\right)$ is a stable pair for a compensator $Z_{c i}$ if and only if $Z_{c i}=\left(L_{i}-D_{r i} X_{i}\right)\left(K_{i}+\right.$ $\left.N_{r i} X_{i}\right)^{-1}$ for some $X_{i}$ over $S$ provided $\left(K_{i}+N_{r i} X_{i}\right)$ is biproper. Also let a left coprime fraction of $Z$ be given by $Q^{-1}\left[R_{1} \cdots R_{N}\right]$ where $Q \in \boldsymbol{S}^{p \times p}, R_{i} \in S^{p \times r_{i}}, i \in \boldsymbol{N}$. Define $P_{i} \in S^{p_{i} \times p}, i \in N$ as follows: $\left[P_{1}^{\prime} \cdots P_{N}^{\prime}\right]^{\prime}=I_{p}$. Letting $Z_{c 0}:=\operatorname{diag}\left\{L_{1} K_{1}^{-1}, \cdots, L_{N} K_{N}^{-1}\right\}$, define $P=\left[0_{p} \mid I_{p}\right]$

$$
\begin{aligned}
Q_{11} & =\left[\begin{array}{ccccc}
Q & R_{1} L_{1} & R_{2} L_{2} & \cdots & R_{N} L_{N} \\
-P_{1} & K_{1} & 0 & \cdots & 0 \\
-P_{2} & 0 & K_{2} & \cdots & 0 \\
\vdots & \vdots & \vdots & \ddots & \vdots \\
-P_{N} & 0 & 0 & \cdots & K_{N}
\end{array}\right], \\
R & =\left[\begin{array}{cccc}
-R_{1} D_{r 1} & -R_{2} D_{r 2} & \cdots & -R_{N} D_{r N} \\
N_{r 1} & 0 & \cdots & 0 \\
0 & N_{r 2} & \cdots & 0 \\
\vdots & \vdots & \ddots & \vdots \\
0 & 0 & \cdots & N_{r N}
\end{array}\right] .
\end{aligned}
$$

Various coprimeness relations yield that $\bar{Z}\left(Z_{c 0}\right):=P Q_{11}^{-1} R$ is a bicoprime fraction where the nonsingularity of $Q_{11}$ is ensured by the fact that $L_{i}, i \in N$ are strictly proper. There is no simple interpretation for $\bar{Z}\left(Z_{c 0}\right)$ in terms of the plants $Z, T_{i}$, and the initial decentralized controller $Z_{\mathrm{c} 0}$. The unstable poles of $\bar{Z}\left(Z_{c 0}\right)$ (or the unstable zeros of $\operatorname{det}\left(Q_{11}\right)$ ), however, are simply the unstable eigenvalues of the closed-loop system obtained by the application of $Z_{c 0}$ to $Z$. The following theorem yields a solution to DCSP.

Theorem 6: The problem DCSP is solvable for $Z$ and $T_{i}, i \in \boldsymbol{N}$ if and only if DSSP for the plant $\bar{Z}\left(Z_{c 0}\right)$ is solvable.

We omit the proof of Theorem 6 as it is straightforward. It should be noted that if $Z$ satisfies A1) then there exists an initial decentralized controller $Z_{\mathrm{c} 0}$ for $Z$ which satisfies that $\bar{Z}\left(Z_{c 0}\right)$ is strongly connected. Moreover, if $Z$ satisfies both A1) and A2) then there exists an initial decentralized controller $Z_{c 0}$ for $Z$ satisfying that $\bar{Z}\left(Z_{c 0}\right)$ is strongly connected, and $\operatorname{rank} \bar{Z}_{i j} \geq 2$ or rank $\bar{Z}_{j i} \geq 2, \forall i, j \in N, i \neq j$ where $\bar{Z}_{i j} \in$ $\boldsymbol{P}^{p_{i} \times r_{j}}$ denotes the $(i, j)$ th submatrix of $\bar{Z}\left(Z_{c 0}\right), i, j \in N$.

\section{CONCluding Remarks}

- Since the unstable blocking zeros of a (centralized) system remain invariant in the closed-loop transfer matrix under stabilizing feedback, every unstable decentralized blocking zero is a blocking zero of every control channel in the closed-loop system resulting from any decentralized stabilizing feedback.

- DCSP is solved by transforming it to a DSSP on a subsidiary plant. This causes that the solvability conditions for DCSP are given in terms of a transformed plant where the original system data is distorted. It is still possible, however, to give various sufficient conditions for the solution of the particular problems P1) and P2) (Section V) in terms of the original system information [25]. 
- A possible application of DCSP is in the expanding system problem [5], [21], where a set of local controllers are sought to stabilize various subsystems so that when these subsystems are interconnected in a prescribed order the internal stability is maintained. Several necessary conditions can be obtained for the solvability of expanding system problem by formulating it as a combination of various decentralized concurrent stabilization problems.

- A numerical computation of decentralized blocking zeros for a given transfer matrix is possible in two alternative ways. First, assuming that a transfer matrix representation is given, we can list the set of (centralized) blocking zeros of each $Z_{i j}$ and try all possible permutations of $N$ to check if (5) is possible for some $s \in \mathcal{C}_{e}$. If each entry of $Z$ is given as a ratio of coprime polynomials, it is not difficult to compute the set of blocking zeros of any submatrix of $Z$. Second, assuming that a statespace representation or stable coprime representation of $Z$ is given, one can use Remark 1 to identify the set of decentralized blocking zeros. This amounts to computing the rank of a system matrix as in (7) for which there are available computer tools such as XMATH and MATLAB.

\section{REFERENCES}

[1] B. D. O. Anderson and A. Linnemann, "Control of decentralized systems with distributed controller complexity," IEEE Trans. Automat. Contr., vol. AC-32, pp. 625-629, 1987.

[2] E. H. Bristol, "Pinned zeros: An introduction to multivariable zeros and dynamic interaction analysis," ISA Chempid Symposium, Newark, 1980.

[3] J. P. Corfmat and A. S. Morse, "Decentralized control of linear multivariable systems," Automatica, vol. 8, pp. 479-495, 1976.

[4] R. Date and J. Chow, "Decentralized stable factors and parametrization of decentralized controllers," in Proc. Amer. Contr. Conf., Boston, MA, 1991, pp. 904-909.

[5] E. J. Davison and Ü. Özgüner, "The expanding system problem," Sys. Cont. Lett., vol. 1, pp. 255-260, 1982.

[6] C. A. Desoer and N. Gündes, "Algebraic theory of two channel decentralized control systems," in Proc. Amer. Contr. Conf., Atlanta, GA, 1988, pp. 1510-1515.

[7] _ "Bicoprime factorizations of the plant and their relation to rightand left-coprime factorizations," IEEE Trans. Automat. Contr., vol. 33 , pp. $672-676,1988$.

[8] P. G. Ferreira, "The servomechanism problem and the method of the state-space in the frequency domain," Int. J. Contr., vol. 23, pp. 245-255, 1976.

[9] P. G. Ferreira and S. P. Bhattacharyya, "On blocking zeros," IEEE Trans. Automat. Contr., vol. 22, pp. 258-259, 1977.

[10] N. Gündeş and C. A. Desoer, Algebraic Theory of Linear Feedback Systems with Full and Decentralized Compensators. Berlin: SpringerVerlag, 1990.

[11] V. Manousiouthakis, "On the parametrization of all decentralized stabilizing controllers," in Proc. Amer. Contr. Conf., Pittsburgh, PA, 1991, pp. 2108-2111.

[12] M. Morari and E. Zafiriou, Robust Process Control. Englewood Cliffs, NJ: Prentice-Hall, 1989.

[13] A. B. Özgüler, "Decentralized control: A stable proper fractional approach," IEEE Trans. Automat. Contr., vol. 35, pp. 1109-1117, 1990.

[14] Y. Ohta, D. D. Šiljak, and T. Matsumoto, "Decentralized control using quasi-block diagonal dominance of transfer function matrices," IEEE Trans. Automat. Contr., vol. AC-31, pp. 420-429, 1986.

[15] A. B. Özgüler and K. A. Ünyelioğlu, "Decentralized strong stabilization problem," in Proc. Amer. Contr. Conf., Chicago, IL, 1992, pp. 3294-3298.

[16] Ü. Özgüner, L. C. Lee, and E. J. Davison, "Minimal realization of a class of decentralized systems," in Proc. Amer. Contr. Conf., San Francisco, CA, 1983, pp. 40-45.

[17] H. H. Rosenbrock, "Design of multivariable control systems using the inverse Nyquist array," Proc. IEE, vol. 116, pp. 1929-1936, 1969.

[18] $\frac{1}{1970}$, State-Space and Multivariable Theory. New York: Wiley,
[19] M. G. Singh, Decentralized Control. Amsterdam: North-Holland, 1981

[20] H. Tamura and T. Yoshikawa, Large-Scale Systems Control and Decision Making. New York: Marcel Dekker, 1990.

[21] X.-L. Tan and M. Ikeda, "Decentralized stabilization for expanding structure of large-scale systems," IEEE Trans. Automat. Contr., vol. 35, pp. 644-651, 1990.

[22] K. A. Ünyelioğlu, "Decentralized blocking zeros in the control of large scale systems," Ph.D. dissertation, Bilkent University, Ankara, Turkey, 1992.

[23] K. A. Ünyelioğlu and A. B. Özgüler, "Decentralized stabilization: Characterization of all solutions and genericity aspects," Int. J. Contr., vol. 55, pp. 1381-1403, 1992.

[24] back interconnected systems," IEEE Trans. Automat. Contr., vol. 37, pp. 1119-1132, 1992.

[25] _ "Decentralized blocking zeros-Part II: A unifying approach to decentralized synthesis problems," in Proc. 1993 Amer. Contr. Conf., San Francisco, CA, 1994, pp. 2745-2749.

[26] K. A. Ünyelioğlu and Ü. Özgüner, " $H_{\infty}$ sensitivity minimization using decentralized feedback: 2-input 2-output systems," Syst. Contr. Lett., vol. 22, pp. 99-109, 1994.

[27] K. A. Ünyelioğlu A. B. Özgüler, and Ü. Özgüner, "Decentralized blocking zeros," Tech. Rep., Ohio State Univ., 1994.

[28] R. J. Veillette, J. V. Medanić, and W. R. Perkins, "Design of reliable control systems," IEEE Trans. Automat. Contr., vol. 37, pp. 290-304, 1992.

[29] M. Vidyasagar, Control System Synthesis: A Factorization Approach. Cambridge, MA: MIT Press, 1985.

[30] M. Vidyasagar and N. Viswanadham, "Algebraic design techniques for reliable stabilization," IEEE Trans. Automat. Contr., vol. 27, pp. 1085-1095, 1982

[31] S. H. Wang and E. J. Davison, "On the stabilization of decentralized control systems," IEEE Trans. Automat. Contr., vol. 18, pp. 473-478, 1973.

[32] D. C. Youla, J. J. Bongiorno, Jr., and C. N. Lu, "Single-loop feedback feedback stabilization of linear multivariable plants," Automatica, vol. 10, pp. 159-173, 1974.

[33] G. Zames and D. Bensoussan, "Multivariable feedback, sensitivity, and decentralized control," IEEE Trans. Automat. Contr., vol. AC-28, pp. 1030-1035, 1983.

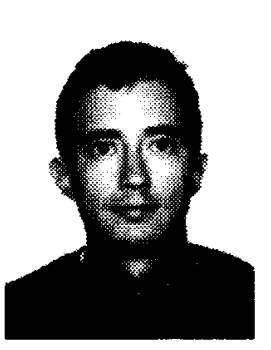

Konur A. Ünyelioğlu received the B.S. degree from the Middle East Technical University, Ankara, Turkey, and the M.Sc. and Ph.D. degrees from Bilkent University, Ankara, Turkey, in 1986, 1988, and 1992, respectively, all in electrical and electronics engineering.

$\mathrm{He}$ is currently with the Ohio State University, Columbus, where he holds a Research Associate position at the Department of Electrical Engineering. His current research interests are in the field of large scale systems and automotive control.

A. Bülent Özgüler received the B.Sc. and M.Sc. degrees from the Middle East Technical University, Ankara, Turkey, in 1976 and 1978, respectively, and the Ph.D. degree from the Center for Mathematical System Theory, University of Florida, Gainesville, in 1982, all in electrical engineering.

He was a Researcher at the Applied Mathematics division of Marmara Research Institute, Kocaeli, Turkey, from 1983 to 1986 . He is currently with the Department of Electrical and Electronics Engineering, Bilkent University, Ankara, Turkey. His research interests are in the field of mathematical control theory.

Dr. Özgüler is a recipient of the 1985 Sedat Simavi Foundation Award for applied sciences and of a research prize of the Scientific and Technological Research Council of Turkey (TÜBITTAK) in 1987. 


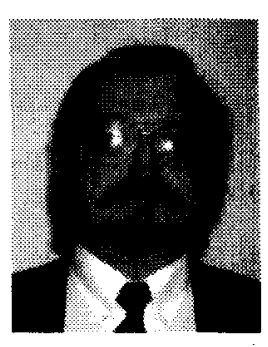

Ümit Özgüner received the Ph.D. degree from the University of Illinois, Urbana, in 1975.

He has held research and teaching positions at IBM T. J. Watson Research Center, University of Toronto, and Istanbul Technical University. He has been with the Ohio State University, Colimbus, since 1981 where he is presently Professor of Electrical Engineering and Director of Center for Intelligent Transportation Research. His areas of research interest are in decentralized and hierarchical control for large scale systems and mechatronic systems in general and applied automotive control, flexible structure control and IVHS $\mathrm{He}$ is the author of over 200 publications which have appeared in journals, as book chapters and in conference proceedings.

Dr. Özgüner the is Technical Committee Chair on Intelligent Control for the IEEE Control Society, represents the Control Society in the IEEE TAB Intelligent Transportation Systems Committee, and is chairing the Ohio Aerospace Institute Technet on Control. He has participated in the organization of many conferences and has been the Technical Program Cochair of the 1993 and General Chair of the 1994 International Symposium on Intelligent Control. 